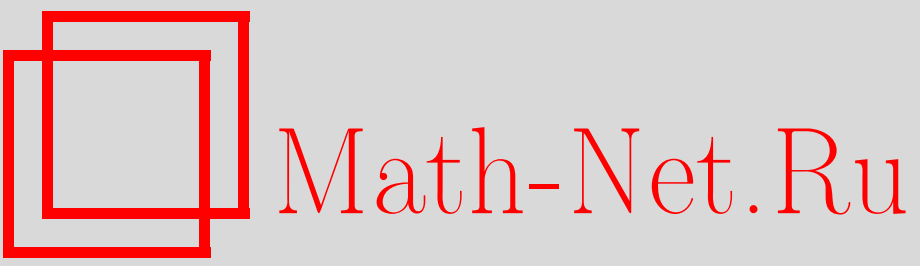

А. А. Белавин, А. В. Одесский, Р. А. Усманов, Новые соотношения в алгебре $Q$-операторов Бакстера, TMФ, 2002, том 130, номер 3, 383-413

DOI: https://doi.org/10.4213/tmf308

Использование Общероссийского математического портала Math-Net.Ru подразумевает, что вы прочитали и согласны с пользовательским соглашением

http://www.mathnet.ru/rus/agreement

Параметры загрузки:

IP: 18.208 .226 .222

26 апреля 2023 г., 18:05:35 
ТЕОРЕТИЧЕСКАЯ

И МАТЕМАТИЧЕСКАЯ

ФИЗИКА

Том 130, № 3

март, 2002

(С) 2002 г. $\quad$ А.А. Белавин*, А.В. Одесский*, Р.А. Усманов*

\section{НОВЫЕ СООТНОШЕНИЯ В АЛГЕБРЕ Q-ОПЕРАТОРОВ БАКСТЕРА}

Рассмотрены неприводимые циклические представления алгебры матриц монодромии, соответствующие $R$-матрице шестивершинной модели. В корнях из единицы $Q$ оператор Бакстера может быть представлен как след тензорного произведения $L$-операторов, отвечающих одному из таких представлений. Для него справедливо $T Q$-уравнение. Найдена новая алгебраическая структура, образуемая этими $L$-операторами и, как следствие, $Q$-операторами.

\section{1. ВВЕДЕНИЕ}

В работах [1] Бакстер ввел $Q$-оператор, которьй он использовал для решения восьмивершинной модели. $Q$-операторы образуют семейство, коммутируюшее с семейством трансфер-матриц $T(u)$, причем имеет место $T Q$-уравнение, связываюшее два семейства между собой и являюшееся ключевым для решения модели.

В работе [2] (см. также [3], [4]) были найдены выражения для больцмановских весов киральной модели Поттса, являюшихся решением уравнения "звезда-треугольник". $R$-матрица модели $S$ может быть представлена в виде произведения четырех таких больцмановских весов.

Алгебраическая структура $Q$-оператора в частном случае шестивершинной модели и связь между ним и $R$-матрицей киральной модели Поттса были найдены в работе Бажанова и Строганова [5], которые в корнях $N$-й степени из единицы ( $N$ - простое число) нашли $N$-мерное циклическое представление $\mathcal{L}$ алгебры Янга-Бакстера, связанной с $R$-матришей обычной шестивершинной модели. $C л е д$ по $N$-мерному квантовому пространству тензорного произведения $L$-операторов обладает свойствами $Q$-оператора. В частности, он удовлетворяет $T Q$-уравнению.

В работе Тарасова [6] были исследованы неприводимые циклические представления алгебры матриц монодромии, соответствуюшей $R$-матрице шестивершинной модели в корнях из единицы.

${ }^{*}$ Институт теоретической физики им. Л. Д. Ландау РАН, г. Черноголовка, Московская обл., Россия 
В последнее время $Q$-оператор опять привлек повышенное внимание в связи с тем, что он является квантовым аналогом преобразования Беклунда, как это было показано в работах [7]-[10] для некоторых моделей статистической физики.

В упомянутой уже работе [5] $R$-матрица $S$ киральной модели Поттса была получена как оператор, сплетающий тензорные произведения двух циклических представлений алгебры матриц монодромии, причем последние перемножаются сначала в одном порядке, а затем в обратном.

Четыре сомножителя, образуюшие $R$-матрицу $S$, являются сплетающими операторами для специальных случаев некоторых элементарных изоморфизмов циклических представлений алгебры $L$-операторов и их тензорных произведений.

В данной работе мы выясняем, при каких условиях в одном случае два циклических представления, а в другом - два тензорных произведения пары циклических представлений алгебры матриц монодромии являются эквивалентными, и находим соответствующие сплетаюшие операторы. Последние являются обобшением известных вершинных весов киральной модели Поттса и удовлетворяют некоторой модификации уравнений “звезда-треугольник”.

План работы следующий. Во втором разделе мы вводим циклические представления алгебры $L$-операторов. В третьем - обсуждаем разные варианты записи циклических представлений. В разделе 4 выводится $T Q$-уравнение. В пятом разделе рассмотрены некоторые частные случаи элементарных изоморфизмов, действующих на циклических представлениях алгебры матриц монодромии. В шестом разделе мы выводим их в общем случае и показьваем, что сплетающие операторы этих элементарных изоморфизмов удовлетворяют обобщенному уравнению “звезда-треугольник” киральной модели Поттса. В разделе 7 выписываются соотношения в алгебре $Q$-операторов. В восьмом разделе обсуждаются перспективы будущих исследований. В приложениях дается вывод некоторых формул, встречающихся в тексте.

\section{2. ЦИКЛИЧЕСКИЕ ПРЕДСТАВЛЕНИЯ АЛГЕБРЫ ЯНГА-БАКСТЕРА}

Следуя работе [6], введем определение $R$-матрицы

$$
R(u)=\left(\begin{array}{cccc}
1-u \omega & 0 & 0 & 0 \\
0 & \omega(1-u) & u(1-\omega) & 0 \\
0 & 1-\omega & 1-u & 0 \\
0 & 0 & 0 & 1-u \omega
\end{array}\right)
$$

Она связана с алгеброй $U_{\mathrm{q}}\left(s l_{2}\right)[11]-[13]$ и может быть получена из $R$-матрицы обычной шестивершинной модели при помощи простого преобразования (см. раздел 3$)$. Для краткости обозначим $\mathcal{M}=\operatorname{End} \mathbb{C}^{2}$, так что $R(u) \in \mathcal{M} \otimes \mathcal{M}$.

Алгебра матриш монодромии $\mathcal{A}$ - это алгебра с генераторами $A(u), B(u), C(u), D(u)$, 
$H, H^{-1}$ и соотношениями

$$
\begin{gathered}
R(u) \stackrel{1}{L}(u v)_{2}^{2}(v)=\stackrel{2}{L}(v) \stackrel{1}{L}(u v) R(u), \\
\widehat{\omega} \otimes H, L(u)]=0, \quad H H^{-1}=H^{-1} H=1, \\
L(u)=\left(\begin{array}{cc}
A(u) & B(u) \\
C(u) & D(u)
\end{array}\right) \in \mathcal{M} \otimes \mathcal{A}, \quad \widehat{\omega}=\operatorname{diag}(1, \omega) .
\end{gathered}
$$

Цифры 1 и 2 над $L$ обозначают двумерное пространство, по которому соответствующий $L$-оператор умножается на $R$-матрищу. При этом оба $L$-оператора действуют в одном и том же квантовом пространстве.

Как указано в [6], в алгебре $\mathcal{A}$ можно определить копроизведение $\Delta$ :

$$
\begin{gathered}
\Delta(L(u))=L_{1}(u) L_{2}(u) \in \mathcal{M} \otimes \mathcal{A} \otimes \mathcal{A}, \\
\Delta(H)=H \otimes H .
\end{gathered}
$$

Нижние индексы 1 и 2 обозначают квантовые пространства, в которых действуют соответствующие $L$-операторы. При этом сами $L$-операторы, рассматриваемые как двумерные матрицы (каждый матричный элемент является оператором в квантовом пространстве), перемножаются между собой по обычным правилам умножения матриц. Тем самым тензорное произведение представлений алгебры $\mathcal{A}$ также является представлением $\mathcal{A}$.

Определим квантовый детерминант

$$
\operatorname{det}_{q} L(u)=D(u) A\left(u \omega^{-1}\right)-C(u) B\left(u \omega^{-1}\right)
$$

Можно показать, что $H^{-1} \operatorname{det}_{q} L(u)$ является центральным элементом алгебры $\mathcal{A}$. Здесь и далее будем считать, что $\omega^{N}=1$. В этом случае, как показано в [6], центр алгебры $\mathcal{A}$ увеличивается, а именно, центральными становятся операторы

$$
\langle\mathcal{O}\rangle(u)=\prod_{k=0}^{N-1} \mathcal{O}\left(u \omega^{k}\right), \quad \mathcal{O}=A, B, C, D
$$

и, таким образом, можно определить матрицу элементов центра

$$
\langle L\rangle=\left(\begin{array}{cc}
\langle A\rangle & \langle B\rangle \\
\langle C\rangle & \langle D\rangle
\end{array}\right)
$$

Можно показать [6], что для $L=L_{1} L_{2}$ имеет место равенство

$$
\Delta(\langle L\rangle)=\left\langle L_{1}\right\rangle\left\langle L_{2}\right\rangle, \quad\left\langle\operatorname{det}_{q} L\right\rangle=\operatorname{det}\langle L\rangle
$$

2 Теоретическая и математическая физика, т. 130, № 3, 2002 г. 
$N$-мерное представление $\pi$ алгебры $\mathcal{A}$ определяется следующим образом [6]:

$$
\begin{aligned}
L\left(u, p_{1}, p_{2}\right) & =\left(\begin{array}{cc}
c_{1} c_{2} Z-b_{1} b_{2} u & -u\left(b_{1} d_{2}-c_{1} a_{2} Z\right) X \\
X^{-1}\left(d_{1} b_{2}-a_{1} c_{2} Z\right) & d_{1} d_{2}-a_{1} a_{2} \omega u Z
\end{array}\right), \\
H_{\pi} & =h Z, \quad p_{i}=\left(a_{i}, b_{i}, c_{i}, d_{i}\right), \quad i=1,2 .
\end{aligned}
$$

Действие операторов $X, Z$ для стандартного базиса в $\mathbb{C}^{N}$ дается формулами

$$
Z|k\rangle=\omega^{k}|k\rangle, \quad X|k\rangle=|k+1\rangle, \quad k=0, \ldots, N-1, \quad|N\rangle \equiv|0\rangle .
$$

Мы также имеем

$$
\left\langle L\left(p_{1}, p_{2}\right)\right\rangle(v)=\left(\begin{array}{cc}
c_{1}^{N} c_{2}^{N}-b_{1}^{N} b_{2}^{N} v & -v\left(b_{1}^{N} d_{2}^{N}-c_{1}^{N} a_{2}^{N}\right) \\
d_{1}^{N} b_{2}^{N}-a_{1}^{N} c_{2}^{N} & d_{1}^{N} d_{2}^{N}-a_{1}^{N} a_{2}^{N} v
\end{array}\right) .
$$

Хотя в формулы (4) входят восемь параметров (помимо спектрального), $N$-мерное представление зависит только от шести из них, поскольку при замене

$$
\begin{aligned}
a_{1} & \rightarrow \lambda a_{1}, & a_{2} & \rightarrow \lambda^{-1} a_{2}, \\
c_{1} & \rightarrow \lambda c_{1}, & c_{2} & \rightarrow \lambda^{-1} c_{2}, \\
b_{1} \rightarrow b_{1}, & b_{2} & \rightarrow b_{2}, & d_{1} \rightarrow d_{1}, \quad d_{2} \rightarrow d_{2},
\end{aligned}
$$

где $\lambda$ - произвольное число, оператор $L\left(u, p_{1}, p_{2}\right)$ не меняется. То же верно и для замены

$$
\begin{aligned}
b_{1} & \rightarrow \lambda b_{1}, \quad b_{2} \rightarrow \lambda^{-1} b_{2}, \\
d_{1} & \rightarrow \lambda d_{1}, \quad d_{2} \rightarrow \lambda^{-1} d_{2}, \\
a_{1} \rightarrow a_{1}, \quad a_{2} & \rightarrow a_{2}, \quad c_{1} \rightarrow c_{1}, \quad c_{2} \rightarrow c_{2} .
\end{aligned}
$$

Кроме того, с точностью до классов проективной эквивалентности $L$-оператор зависит только от четырех параметров:

$$
L\left(\lambda p_{1}, p_{2}\right)=\lambda L\left(p_{1}, p_{2}\right), \quad L\left(p_{1}, \mu p_{2}\right)=\mu L\left(p_{1}, p_{2}\right),
$$

где $\lambda, \mu$ - произвольные числа.

Два представления $L_{1}\left(u, p_{1}, p_{2}\right) L_{2}\left(u, p_{3}, p_{4}\right)$ и $L_{2}\left(u, p_{3}, p_{4}\right) L_{1}\left(u, p_{1}, p_{2}\right)$ эквивалентны тогда и только тогда, когда можно выбрать $p_{i}(i=1,2,3,4)$ так, что они удовлетворяют условиям

$$
\frac{a_{i}^{N} \pm b_{i}^{N}}{c_{i}^{N} \pm d_{i}^{N}}=\lambda_{ \pm}
$$

где $\lambda_{ \pm}$не зависят от $i$ (см. приложение Б). Сплетаюший оператор, определяемый уравнением

$$
\mathbf{S}\left(p_{1}, p_{2}, p_{3}, p_{4}\right) L_{1}\left(u, p_{1}, p_{2}\right) L_{2}\left(u, p_{3}, p_{4}\right)=L_{1}\left(u, p_{3}, p_{4}\right) L_{2}\left(u, p_{1}, p_{2}\right) \mathbf{S}\left(p_{1}, p_{2}, p_{3}, p_{4}\right),
$$
может быть явно выражен через больцмановские веса киральной модели Поттса $W_{p q}$, $\bar{W}_{p q}$ :

$$
\begin{gathered}
\mathbf{S}\left(p_{1}, p_{2}, p_{3}, p_{4}\right)=\mathbf{P} F\left(p_{1}, p_{4} ; X_{1} X_{2}^{-1}\right) G\left(p_{1}, p_{3} ; Z_{1}\right) G\left(p_{2}, p_{4} ; Z_{2}\right) F\left(p_{2}, p_{3} ; X_{1} X_{2}^{-1}\right), \\
G\left(p, q ; \omega^{k}\right)=W_{p q}(k), \quad F\left(p, q ; \omega^{k}\right)=\sum_{l=1}^{N} \omega^{k l} \bar{W}_{p q}(l),
\end{gathered}
$$

где $\mathbf{P}$ - обычный оператор перестановки, меняющий пространства 1 и 2 местами. 


\section{3. ЦИКЛИЧЕСКИЕ ПРЕДСТАВЛЕНИЯ АЛГЕБРЫ ЯНГА-БАКСТЕРА В ФОРМЕ БАЖАНОВА-СТРОГАНОВА}

Наряду с введенными в предыдушей части $L$-операторами можно рассматривать их вариант, связанный с другим выбором $R$-матришы.

Рассмотрим обычную $R$-матрицу модели льда

$$
R_{\text {ice }}(x)=\left[\begin{array}{cccc}
x \omega_{1}-x^{-1} \omega_{1}^{-1} & 0 & 0 & 0 \\
0 & x-x^{-1} & \omega_{1}-\omega_{1}^{-1} & 0 \\
0 & \omega_{1}-\omega_{1}^{-1} & x-x^{-1} & 0 \\
0 & 0 & 0 & x \omega_{1}-x^{-1} \omega_{1}^{-1}
\end{array}\right]
$$

и соответствуюшие ей соотношения в алгебре Янга-Бакстера:

$$
R_{\text {ice }}(x) \stackrel{1}{L} \text { ice }(x y) \stackrel{2}{L} \text { ice }(y)=\stackrel{2}{L_{\text {ice }}}(y) \stackrel{1}{L} \text { ice }(x y) R_{\text {ice }}(x) .
$$

$N$-мерное представление алгебры (9) можно записать следуюшим образом:

$$
L_{\text {ice }}\left(y, p_{1}, p_{2}\right)=\left(\begin{array}{cc}
y^{-1} c_{1} c_{2} Z_{1}-b_{1} b_{2} y Z_{1}^{-1} & -\left(b_{1} d_{2} Z_{1}^{-1}-c_{1} a_{2} Z_{1}\right) X \\
\omega_{1} X^{-1}\left(d_{1} b_{2} Z_{1}^{-1}-a_{1} c_{2} Z_{1}\right) & y^{-1} d_{1} d_{2} Z_{1}^{-1}-a_{1} a_{2} \omega_{1}^{2} y Z_{1}
\end{array}\right),
$$

где операторы $Z_{1}, X$ удовлетворяют соотношениям

$$
Z_{1}^{N}=1, \quad X^{N}=1, \quad Z_{1} X=\omega_{1} X Z_{1} .
$$

Сделаем замену

$$
\begin{gathered}
R_{12} \rightarrow C_{1}^{-1}(x y) C_{2}^{-1}(y) R_{12} C_{2}(y) C_{1}(x y), \quad L_{1}(x y) \rightarrow C_{1}^{-1}(x y) L_{1}(x y) C_{1}(x y), \\
L_{2}(y) \rightarrow C_{2}^{-1}(y) L_{2}(y) C_{2}(y),
\end{gathered}
$$

где

$$
C(y)=\left(\begin{array}{ll}
1 & 0 \\
0 & y
\end{array}\right)
$$

а индекс у матришы $C$ показывает, в каком из двух пространств она действует. В результате такой замены уравнение $R L L=L L R$ по-прежнему будет иметь место. При этом

$$
\mathcal{R}(x)=\left(\begin{array}{cccc}
x \omega_{1}-x^{-1} \omega_{1}^{-1} & 0 & 0 & 0 \\
0 & x-x^{-1} & x\left(\omega_{1}-\omega_{1}^{-1}\right) & 0 \\
0 & x^{-1}\left(\omega_{1}-\omega_{1}^{-1}\right) & x-x^{-1} & 0 \\
0 & 0 & 0 & x \omega_{1}-x^{-1} \omega_{1}^{-1}
\end{array}\right)
$$

а $L$-оператор дается формулой

$$
\mathcal{L}\left(y, p_{1}, p_{2}\right)=\left(\begin{array}{cc}
y^{-1} c_{1} c_{2} Z_{1}-b_{1} b_{2} y Z_{1}^{-1} & -y\left(b_{1} d_{2} Z_{1}^{-1}-c_{1} a_{2} Z_{1}\right) X \\
\omega_{1} y^{-1} X^{-1}\left(d_{1} b_{2} Z_{1}^{-1}-a_{1} c_{2} Z_{1}\right) & y^{-1} d_{1} d_{2} Z_{1}^{-1}-a_{1} a_{2} \omega_{1}^{2} y Z_{1}
\end{array}\right) .
$$


Именно последний $L$-оператор был найден в работе [5], и именно его мы будем называть циклическим представлением алгебры матриц монодромии в форме Бажанова-Строганова.

Домножим теперь $L(y)$ на $y Z_{1}$ и введем следуюшие обозначения:

$$
v=y^{2}, \quad Z=Z_{1}^{2}, \quad \omega=\omega_{1}^{2} .
$$

Тогда получим используемый нами оператор (4). При этом, чтобы уравнение $R L L=$ $L L R$ было по-прежнему верным, необходимо домножить $R$-матрицу с двух сторон следуюшим образом:

$$
R(u)=-\omega_{1} x K_{1} \mathcal{R}(x) K_{2}^{-1},
$$

где

$$
u=x^{2}, \quad K=\omega_{1}^{\left(\sigma^{z}-1\right) / 2}=\left(\begin{array}{cc}
1 & 0 \\
0 & \omega_{1}^{-1}
\end{array}\right),
$$

а нижний индекс у матрицы $K$ обозначает пространство, в котором она действует. Матрица $R(u)$, как нетрудно видеть, совпадает с (1), а $L$-оператор - с $L$-оператором (4).

Подчеркнем, что в дальнейшем мы везде будем обозначать циклические представления в форме Тарасова буквой $L$, а циклические представления в форме Бажанова-Строганова - буквой $\mathcal{L}$. Матричные элементы $L$-операторов будем обозначать соответственно $L_{i \alpha}^{j \beta}$ и $\mathcal{L}_{i \alpha}^{j \beta}, i, j=0,1, \alpha, \beta=0, \ldots, N-1$.

\section{Q-ОПЕРАТОР И ТQ-УРАВНЕНИЕ}

Трансфер-матрища, построенная с помошью $\mathcal{L}(u)$,

$$
\mathcal{Q}(u)=\operatorname{tr}_{0} \mathcal{L}_{10}(u) \mathcal{L}_{20}(u) \ldots \mathcal{L}_{n 0}(u),
$$

где след берется по $N$-мерному пространству, обладает одним очень важным свойством. Она удовлетворяет $T Q$-уравнению, что позволяет говорить о ней как о $Q$-операторе Бакстера [5]. Покажем это.

Рассмотрим уравнение

$$
\mathcal{R}_{i_{1} i_{2}}^{j_{1} j_{2}}(u) \mathcal{L}_{j_{1} \alpha}^{k_{1} \beta}(u v) \mathcal{L}_{j_{2} \beta}^{k_{2} \gamma}(v)=\mathcal{L}_{i_{2} \alpha}^{j_{2} \beta}(u v) \mathcal{L}_{i_{1} \beta}^{j_{1} \gamma}(v) \mathcal{R}_{j_{1} j_{2}}^{k_{1} k_{2}}(u)
$$

Его можно изобразить графически так, как показано на рис. 1. При фиксированных индексах $i_{1}, k_{1}$ обе части уравнения (12) являются операторами в тензорном произведении $\mathbb{C}^{2} \times \mathbb{C}^{N}$. Подействуем этими операторами на вектор $\psi_{k_{2} \gamma}$, принадлежащий ядру оператора $\mathcal{L}_{23}(v)$, т.е. на вектор, удовлетворяющий равенству

$$
\mathcal{L}_{j_{2} \beta}^{k_{2} \gamma}(v) \psi_{k_{2} \gamma}=0
$$

Ненулевое ядро у $\mathcal{L}_{23}(v)$ имеется только при некоторых специальных значениях $v=v_{*}$ : $v_{*}^{2}=c_{1} d_{1} / a_{1} b_{1}$ или $v_{*}^{2}=c_{2} d_{2} / a_{2} b_{2}$. Из рис. 1 видно, что ядро оператора $\mathcal{L}_{23}\left(v_{*}\right)$ представляет собой подпространство, инвариантное относительно тензорного произведения 
$\mathcal{L}_{13}\left(u v_{*}\right) \mathcal{R}_{12}(u)$. Дополнение ядра, рассматриваемое как фактор-пространство, в этом случае также является инвариантным подпространством. Поэтому матрица оператора $\mathcal{L}_{13}\left(u v_{*}\right) \mathcal{R}_{12}(u)$ имеет блочно-диагональный вид

$$
\mathcal{L}_{13}\left(u v_{*}\right) \mathcal{R}_{12}(u)=\left(\begin{array}{cc}
P_{1} & * \\
0 & P_{2}
\end{array}\right),
$$

где все блоки суть $N$-мерные квадратные матрицы, а звездочкой обозначены несущественные для нас матричные элементы. Мыздесь ввели следуюший способ упорядочения векторов базиса: первые $N$ векторов образуют ядро, а остальные $N$ - его дополнение.

$$
\mathcal{R}_{i_{1} i_{2}}^{j_{1} j_{2}}(u)=\frac{i_{1} \underbrace{f_{j_{1}}^{j_{2}}}_{i_{i_{2}}}}{j_{2}} \quad \mathcal{L}_{i \alpha}^{j \beta}(v)=\stackrel{i}{v_{\| \alpha}} \|_{\|}^{\beta}
$$

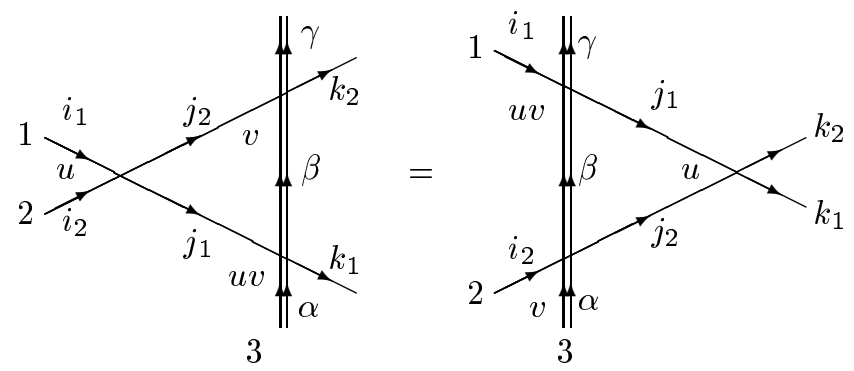

Рис. 1

Пусть при некоторых значениях параметров $\mathcal{L}\left(v_{*}\right)$ имеют место равенства

$$
P_{1}=\phi_{1} \mathcal{L}\left(u v_{*} \lambda\right), \quad P_{2}=\phi_{2} \mathcal{L}\left(u v_{*} \lambda^{-1}\right) .
$$

Тогда после перемножения $n$ копий оператора $\mathcal{L}_{13}\left(u v_{*}\right) \mathcal{R}_{12}(u)$ по пространствам 2 и 3 (см. рис. 2) и взятия следа мы получим уравнение

$$
\widetilde{\mathcal{Q}}\left(u v_{*}\right) T(u)=\phi_{1}^{n} \widetilde{\mathcal{Q}}\left(u v_{*} \lambda\right)+\phi_{2}^{n} \widetilde{\mathcal{Q}}\left(u v_{*} \lambda^{-1}\right)
$$

где

$$
\widetilde{\mathcal{Q}}\left(u v_{*}\right)=\operatorname{tr}_{3} \mathcal{L}_{13}\left(u v_{*}\right) \mathcal{L}_{1^{\prime} 3}\left(u v_{*}\right) \ldots \mathcal{L}_{1^{(n)} 3},
$$




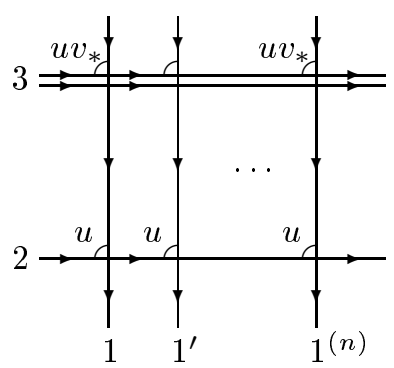

Рис. 2

а $T(u)$ - обычная трансфер-матрица модели льда. Сделав замену $\mathcal{Q}(u)=\widetilde{\mathcal{Q}}\left(u v_{*}\right)$, мы придем к $T Q$-уравнению.

Оказывается, что при том определении оператора $\mathcal{L}(v)$, которое мы имеем, довести изложенную схему до конца не удается, поскольку ни при каком выборе параметров добиться выполнения условий (13) нельзя.

Однако можно так переопределить оператор $\mathcal{L}(v)$, чтобы условие (13), а следовательно, и уравнение (14) действительно имели место [5]. Для этого параметры $p_{1}$ нужно сделать зависящими от спектрального параметра $v$, а параметры $p_{2}$ оставить прежними, т.е.

$$
p_{1}(v)=\left(a_{1} v^{-1}, b_{1}, c_{1}, d_{1} v\right), \quad p_{2}(v)=\left(a_{2}, b_{2}, c_{2}, d_{2}\right) .
$$

В приложении А показано, что $\mathcal{Q}(u)=\mathcal{Q}\left(u, p_{1}(u), p_{2}\right)$ действительно удовлетворяет $T Q$-уравнению:

$$
\mathcal{Q}(u) T(u)=\left(u-u^{-1}\right)^{n} \mathcal{Q}(u \omega)+\left(u \omega-u^{-1} \omega^{-1}\right)^{n} \mathcal{Q}\left(u \omega^{-1}\right) .
$$

Здесь $T(u)$ - обычная трансфер-матрища модели льда.

\section{5. ЭЛЕМЕНТАРНЫЕ ИЗОМОРФИЗМЫ: ЧАСТНЫЙ СЛУЧАЙ}

Рассмотрим два представления алгебры $L$-операторов $L\left(u, p_{1}, p_{2}\right)$ и $L\left(u, p_{2}, p_{1}\right)$. Пусть параметры $p_{1}$ и $p_{2}$ таковы, что оба эти представления эквивалентны. Введем оператор $G$, удовлетворяюший уравнению

$$
G(Z) L\left(u, p_{1}, p_{2}\right)=L\left(u, p_{2}, p_{1}\right) G(Z),
$$

где $G(Z)$ действует в $N$-мерном пространстве.

Теперь рассмотрим два тензорных произведения пары циклических представлений: $L_{1}\left(u, p_{1}, p_{2}\right) L_{2}\left(u, p_{3}, p_{4}\right)$ и $L_{1}\left(u, p_{1}, p_{3}\right) L_{2}\left(u, p_{2}, p_{4}\right)$ (следует обратить внимание на перестановку $\left.p_{2} \leftrightarrow p_{3}\right)$. Пусть параметры $p_{1}, p_{2}, p_{3}, p_{4}$ таковы, что оба представления эквивалентны. Введем оператор $F$, сплетающий их:

$$
F\left(X_{1} X_{2}^{-1}\right) L_{1}\left(u, p_{1}, p_{2}\right) L_{2}\left(u, p_{3}, p_{4}\right)=L_{1}\left(u, p_{1}, p_{3}\right) L_{2}\left(u, p_{2}, p_{4}\right) F\left(X_{1} X_{2}^{-1}\right) .
$$


Здесь $F\left(X_{1} X_{2}^{-1}\right)$ также действует в $N$-мерном пространстве.

Оказывается, условия (5) являются заведомо достаточными для сушествования операторов $G$ и $F$. При их выполнении оба оператора даются следуюшими формулами:

$$
\begin{aligned}
& \frac{G\left(p_{1}, p_{2} ; \omega^{k}\right)}{G\left(p_{1}, p_{2} ; 1\right)}=\prod_{j=1}^{k} \frac{d_{1} b_{2}-a_{1} c_{2} \omega^{j}}{b_{1} d_{2}-c_{1} a_{2} \omega^{j}} \\
& \frac{F\left(p_{1}, p_{2} ; \omega^{k}\right)}{F\left(p_{1}, p_{2} ; 1\right)}=\prod_{j=1}^{k} \frac{\omega a_{1} d_{2}-d_{1} a_{2} \omega^{j}}{c_{1} b_{2}-b_{1} c_{2} \omega^{j}} .
\end{aligned}
$$

Здесь символами $G\left(p_{1}, p_{2} ; \omega^{k}\right)$ и $F\left(p_{1}, p_{2} ; \omega^{k}\right)$ обозначены диагональные матричные элементы $N$-мерных матриц $G\left(p_{1}, p_{2} ; Z\right)$ и $F\left(p_{1}, p_{2} ; X_{1} X_{2}^{-1}\right)$ в соответствуюшем каждой из них собственном базисе (матрицы $G$ и $F$ одновременно не могут быть приведены к диагональному виду).

В следуюшем разделе оба оператора будут вычислены в более общем случае, а сейчас просто отметим, что формулы (17), (18) совпадают с формулами (7).

Сушествование элементарных изоморфизмов $G$ и $F$ объясняет факторизацию $R$-матрицы киральной модели Поттса. Переставляя пары, получим цепочку

$$
\left(p_{1}, p_{2}\right)\left(p_{3}, p_{4}\right) \stackrel{F_{1}}{\rightarrow}\left(p_{1}, p_{3}\right)\left(p_{2}, p_{4}\right) \stackrel{G_{1}, G_{2}}{\longrightarrow}\left(p_{3}, p_{1}\right)\left(p_{4}, p_{2}\right) \stackrel{F_{2}}{\rightarrow}\left(p_{3}, p_{4}\right)\left(p_{1}, p_{2}\right) .
$$

Отсюда факторизация $R$-матрицы становится очевидной.

\section{6. ОБЩИЙ СЛУЧАЙ}

6.1. $G$-оператор. Рассмотрим два представления алгебры $L$-операторов $L\left(u, p_{1}\right.$, $\left.\bar{p}_{1}\right)$ и $L\left(u, p_{2}, \bar{p}_{2}\right)$. Мы хотим выяснить, при каких условиях они эквивалентны, и найти соответствующий сплетающий оператор, который является обобшением оператора $G$, введенного формулой (15). Для простоты будем обозначать этот обобшенный сплетающий оператор той же буквой $G$.

Два указанных представления эквивалентны при выполнении следующих условий (см. приложение B):

$$
\begin{gathered}
a_{1}^{N} \bar{a}_{1}^{N}=a_{2}^{N} \bar{a}_{2}^{N}, \quad b_{1}^{N} \bar{b}_{1}^{N}=b_{2}^{N} \bar{b}_{2}^{N}, \\
\frac{\bar{c}_{1}^{N} \bar{d}_{1}^{N}}{\bar{a}_{1}^{N} \bar{b}_{1}^{N}}=\frac{c_{2}^{N} d_{2}^{N}}{a_{2}^{N} b_{2}^{N}}, \quad \frac{\bar{c}_{2}^{N} \bar{d}_{2}^{N}}{\bar{a}_{2}^{N} \bar{b}_{2}^{N}}=\frac{c_{1}^{N} d_{1}^{N}}{a_{1}^{N} b_{1}^{N}}, \\
\frac{d_{1}^{N} \bar{d}_{1}^{N}}{a_{1}^{N} \bar{a}_{1}^{N}}=\frac{d_{2}^{N} \bar{d}_{2}^{N}}{a_{2}^{N} \bar{a}_{2}^{N}}, \\
d_{1}^{N} \bar{b}_{1}^{N}-a_{1}^{N} \bar{c}_{1}^{N}=d_{2}^{N} \bar{b}_{2}^{N}-a_{2}^{N} \bar{c}_{2}^{N} .
\end{gathered}
$$


Рассмотрим простейший вариант, когда извлечение корня $N$-й степени производится простым зачеркиванием буквы $N$. В результате получим систему уравнений

$$
\begin{gathered}
a_{1} \bar{a}_{1}=a_{2} \bar{a}_{2}, \quad b_{1} \bar{b}_{1}=b_{2} \bar{b}_{2}, \\
\frac{\bar{c}_{1} \bar{d}_{1}}{\bar{a}_{1} \bar{b}_{1}}=\frac{c_{2} d_{2}}{a_{2} b_{2}}, \quad \frac{\bar{c}_{2} \bar{d}_{2}}{\bar{a}_{2} \bar{b}_{2}}=\frac{c_{1} d_{1}}{a_{1} b_{1}}, \\
d_{1} \bar{d}_{1}=d_{2} \bar{d}_{2}, \\
d_{1}^{N} \bar{b}_{1}^{N}-a_{1}^{N} \bar{c}_{1}^{N}=d_{2}^{N} \bar{b}_{2}^{N}-a_{2}^{N} \bar{c}_{2}^{N} .
\end{gathered}
$$

Найдем теперь такой оператор $G$, что

$$
G L\left(u, p_{1}, \bar{p}_{1}\right)=L\left(u, p_{2}, \bar{p}_{2}\right) G .
$$

При вьполнении условий (20) оператор $G$ существует. Покажем это. Воспользуемся следующим анзацем:

$$
G=G(Z)
$$

Получим систему уравнений

$$
\begin{aligned}
& G(Z) A_{1}=A_{2} G(Z), \\
& G(Z) B_{1}=B_{2} G(Z), \\
& G(Z) C_{1}=C_{2} G(Z), \\
& G(Z) D_{1}=D_{2} G(Z) .
\end{aligned}
$$

Выбираем базис $|k\rangle, k=0, \ldots, N-1(\bmod N)$ :

$$
Z|k\rangle=\omega^{k}|k\rangle, \quad X|k\rangle=|k+1\rangle .
$$

Очевидно, в этом базисе матрица $G(Z)$ является диагональной. Найдем ее ненулевые матричные элементы.

Первое уравнение полученной системы принимает вид

$$
G(Z)\left(c_{1} \bar{c}_{1} Z-b_{1} \bar{b}_{1} u\right)=\left(c_{2} \bar{c}_{2} Z-b_{2} \bar{b}_{2} u\right) G(Z) .
$$

Действуя обеими частями этого уравнения на вектор $|k\rangle$ и приравнивая коэффициенты при разных степенях $u$, приходим к следуюшему ограничению на параметры:

$$
c_{1} \bar{c}_{1}=c_{2} \bar{c}_{2}, \quad b_{1} \bar{b}_{1}=b_{2} \bar{b}_{2} .
$$

Аналогично из четвертого уравнения

$$
G(Z)\left(d_{1} \bar{d}_{1}-a_{1} \bar{a}_{1} \omega u Z\right)=\left(d_{2} \bar{d}_{2}-a_{2} \bar{a}_{2} \omega u Z\right) G(Z)
$$


получаем, что

$$
d_{1} \bar{d}_{1}=d_{2} \bar{d}_{2}, \quad a_{1} \bar{a}_{1}=a_{2} \bar{a}_{2}
$$

Второе уравнение

$$
G(Z) X^{-1}\left[d_{1} \bar{b}_{1}-a_{1} \bar{c}_{1} Z\right]=\left[d_{2} \bar{b}_{2}-a_{2} \bar{c}_{2} Z\right] G(Z)
$$

дает

$$
G\left(\omega^{k+1}\right)=\frac{d_{1} \bar{b}_{1}-a_{1} \bar{c}_{1} \omega^{k+1}}{d_{2} \bar{b}_{2}-a_{2} \bar{c}_{2} \omega^{k+1}} G\left(\omega^{k}\right),
$$

где величины $G\left(\omega^{k}\right), k=0, \ldots, N-1$, обозначают диагональные элементы матришы $G(Z)$. Точно так же из третьего уравнения

$$
G(Z)\left[b_{1} \bar{d}_{1}-c_{1} \bar{a}_{1} Z\right] X=\left[b_{2} \bar{d}_{2}-c_{2} \bar{a}_{2} Z\right] X G(Z)
$$

легко получить, что

$$
G\left(\omega^{k+1}\right)=\frac{b_{2} \bar{d}_{2}-c_{2} \bar{a}_{2} \omega^{k+1}}{b_{1} \bar{d}_{1}-c_{1} \bar{a}_{1} \omega^{k+1}} G\left(\omega^{k}\right) .
$$

Поскольку функция $G\left(\omega^{k}\right)$ однозначна,

$$
\left(d_{1} \bar{b}_{1}-a_{1} \bar{c}_{1} \omega^{k+1}\right)\left(b_{1} \bar{d}_{1}-c_{1} \bar{a}_{1} \omega^{k+1}\right)=\left(d_{2} \bar{b}_{2}-a_{2} \bar{c}_{2} \omega^{k+1}\right)\left(b_{2} \bar{d}_{2}-c_{2} \bar{a}_{2} \omega^{k+1}\right) .
$$

Приравнивая здесь коэффициенты при разных степенях $\omega$ и учитывая равенства (21), (22), получим еще одно дополнительное к уже имеющимся условие:

$$
\frac{\bar{c}_{1} \bar{d}_{1}}{\bar{a}_{1} \bar{b}_{1}}+\frac{c_{1} d_{1}}{a_{1} b_{1}}=\frac{\bar{c}_{2} \bar{d}_{2}}{\bar{a}_{2} \bar{b}_{2}}+\frac{c_{2} d_{2}}{a_{2} b_{2}} .
$$

Кроме того, из условия периодичности $G\left(\omega^{N+1}\right)=G(\omega)$ следует, что

$$
d_{1}^{N} \bar{b}_{1}^{N}-a_{1}^{N} \bar{c}_{1}^{N}=d_{2}^{N} \bar{b}_{2}^{N}-a_{2}^{N} \bar{c}_{2}^{N}
$$

Используя калибровочные симметрии $L$-операторов, можно положить

$$
a_{1}=\bar{a}_{2}, \quad b_{1}=\bar{b}_{2}
$$

Тогда из условий (20) непосредственно следуют равенства (21), (22), (25), (26).

Таким образом, матричные элементы $G\left(\omega^{k}\right)$ сушествуют и даются рекуррентным соотношением (23).

Нетрудно переписать $G\left(\omega^{k}\right)$ через $p_{1}, \bar{p}_{1}$. Подставляя

$$
\bar{c}_{2}=\frac{c_{1} \bar{c}_{1}}{c_{2}}
$$


в (26), выражаем $c_{2}$ через $p_{1}, \bar{p}_{1}$ :

$$
c_{2}=\bar{c}_{1} \sqrt[N]{\frac{b_{1}^{N} \bar{d}_{1}^{N}-c_{1}^{N} \bar{a}_{1}^{N}}{d_{1}^{N} \bar{b}_{1}^{N}-a_{1}^{N} \bar{c}_{1}^{N}}}=\Lambda\left(p_{1}, \bar{p}_{1}\right) \bar{c}_{1}
$$

где мы ввели функцию

$$
\Lambda\left(p_{1}, p_{2}\right)=\sqrt[N]{\frac{b_{1}^{N} d_{2}^{N}-c_{1}^{N} a_{2}^{N}}{d_{1}^{N} b_{2}^{N}-a_{1}^{N} c_{2}^{N}}}
$$

Отсюда получаем

$$
\frac{G\left(p_{1}, \bar{p}_{1} ; \omega^{k}\right)}{G\left(p_{1}, \bar{p}_{1} ; 1\right)}=\Lambda\left(p_{1}, \bar{p}_{1}\right)^{k} \prod_{j=1}^{k} \frac{d_{1} \bar{b}_{1}-a_{1} \bar{c}_{1} \omega^{j}}{\bar{d}_{1} b_{1}-\bar{a}_{1} c_{1} \omega^{j}}
$$

Подчеркнем, что $G$ зависит только от $p_{1}, \bar{p}_{1}$.

Итак, полученный оператор $G$ осушествляет изоморфизм двух представлений алгебры матриц монодромии $L\left(u, p_{1}, \bar{p}_{1}\right), L\left(u, p_{2}, \bar{p}_{2}\right)$, причем параметры $p_{2}, \bar{p}_{2}$ выражаются через $p_{1}, \bar{p}_{1}$ следуюшим образом:

$$
\begin{aligned}
a_{2}=\bar{a}_{1}, & \bar{a}_{2}=a_{1}, \\
b_{2}=\bar{b}_{1}, & \bar{b}_{2}=b_{1}, \\
c_{2}=\Lambda\left(p_{1}, \bar{p}_{1}\right) \bar{c}_{1}, & \bar{c}_{2}=\Lambda\left(p_{1}, \bar{p}_{1}\right)^{-1} c_{1}, \\
d_{2}=\Lambda\left(p_{1}, \bar{p}_{1}\right)^{-1} \bar{d}_{1}, & \bar{d}_{2}=\Lambda\left(p_{1}, \bar{p}_{1}\right) d_{1} .
\end{aligned}
$$

Оператор $G$, полученный здесь, является обобшением оператора $G$, фигурируюшего в формулах (7), (15). Для того чтобы перейти к такому частному случаю, необходимо положить $p_{2}=\bar{p}_{1}, \bar{p}_{2}=p_{1}$. При этом на параметры $p_{1}, \bar{p}_{1}$ возникает дополнительное ограничение $\Lambda\left(p_{1}, \bar{p}_{1}\right)=1$.

ЗАмечАниЕ. Мы извлекали корни $N$-й степени простым зачеркиванием буквы $N$. Видимо, обший случай сводится к этому. Полное исследование можно было бы провести следуюшим образом. Чтобы два неприводимых представления были эквивалентными, необходимо, чтобы совпадали их центры. Однако, получая наши условия, мы приравняли не все элементы центров. К системе рассмотренных выше уравнений необходимо добавить условие равенства квантовых детерминантов. Мы не исследовали полностью вопрос, к чему приводит это дополнительное условие. Видимо, его можно использовать для выяснения того, как нужно извлекать корень $N$-й степени. При этом резко ограничивается число возможных вариантов. 
6.2. F-оператор. Рассмотрим два представления алгебры $L$-операторов $L_{1}\left(u, p_{1}\right.$, $\left.\bar{p}_{1}\right) L_{2}\left(u, p_{2}, \bar{p}_{2}\right)$ и $L_{1}\left(u, p_{3}, \bar{p}_{3}\right) L_{2}\left(u, p_{4}, \bar{p}_{4}\right)$. Мы хотим найти условия, при которых эти представления будут эквивалентными, и вычислить соответствующий сплетающий оператор, обобщающий оператор $F$, введенный в формулах $(7),(16)$.

Матрица элементов центра имеет вид

$$
\langle L(u, p, \bar{p})\rangle=\left(\begin{array}{cc}
c^{N} \bar{c}^{N}-b^{N} \bar{b}^{N} u & -u\left(b^{N} \bar{d}^{N}-c^{N} \bar{a}^{N}\right) \\
d^{N} \bar{b}^{N}-a^{N} \bar{c}^{N} & d^{N} \bar{d}^{N}-a^{N} \bar{a}^{N} u
\end{array}\right) .
$$

Необходимым условием эквивалентности двух представлений является совпадение центров этих представлений. Поэтому

$$
\left\langle L_{1}\left(u, p_{1}, \bar{p}_{1}\right)\right\rangle\left\langle L_{2}\left(u, p_{2}, \bar{p}_{2}\right)\right\rangle=\left\langle L_{1}\left(u, p_{3}, \bar{p}_{3}\right)\right\rangle\left\langle L_{2}\left(u, p_{4}, \bar{p}_{4}\right)\right\rangle
$$

откуда также следует, что

$$
\operatorname{det}\left\langle L_{1}\left(u, p_{1}, \bar{p}_{1}\right)\right\rangle \operatorname{det}\left\langle L_{2}\left(u, p_{2}, \bar{p}_{2}\right)\right\rangle=\operatorname{det}\left\langle L_{1}\left(u, p_{3}, \bar{p}_{3}\right)\right\rangle \operatorname{det}\left\langle L_{2}\left(u, p_{4}, \bar{p}_{4}\right)\right\rangle \text {. }
$$

В приложении В показано, что из этих условий получаются следующие соотношения между параметрами (мы не рассматриваем тривиальный случай $p_{3}=p_{1}, \bar{p}_{3}=\bar{p}_{1}, p_{4}=$ $\left.p_{2}, \bar{p}_{4}=\bar{p}_{2}\right)$ :

$$
\begin{array}{cc}
b_{1}^{N} \bar{b}_{1}^{N}=b_{3}^{N} \bar{b}_{3}^{N}, & b_{2}^{N} \bar{b}_{2}^{N}=b_{4}^{N} \bar{b}_{4}^{N}, \\
a_{1}^{N} \bar{a}_{1}^{N}=a_{3}^{N} \bar{a}_{3}^{N}, & a_{2}^{N} \bar{a}_{2}^{N}=a_{4}^{N} \bar{a}_{4}^{N}, \\
d_{1}^{N} \bar{b}_{1}^{N}=d_{3}^{N} \bar{b}_{3}^{N}, & a_{2}^{N} \bar{c}_{2}^{N}=a_{4}^{N} \bar{c}_{4}^{N} \\
\frac{c_{2}^{N} d_{2}^{N}}{a_{2}^{N} b_{2}^{N}}=\frac{\bar{c}_{3}^{N} \bar{d}_{3}^{N}}{\bar{a}_{3}^{N} \bar{b}_{3}^{N},} \quad \frac{c_{1}^{N} d_{1}^{N}}{a_{1}^{N} b_{1}^{N}}=\frac{c_{3}^{N} d_{3}^{N}}{a_{3}^{N} b_{3}^{N}} \\
\frac{\bar{c}_{2}^{N} \bar{d}_{2}^{N}}{\bar{a}_{2}^{N} \bar{b}_{2}^{N}}=\frac{\bar{c}_{4}^{N} \bar{d}_{4}^{N}}{\bar{a}_{4}^{N} \bar{b}_{4}^{N}}, \quad \frac{c_{4}^{N} d_{4}^{N}}{a_{4}^{N} b_{4}^{N}}=\frac{\bar{c}_{1}^{N} \bar{d}_{1}^{N}}{\bar{a}_{1}^{N} \bar{b}_{1}^{N}} \\
b_{2}^{N} \bar{c}_{3}^{N}=c_{2}^{N} \bar{b}_{1}^{N} \frac{b_{2}^{N} \bar{c}_{1}^{N}-\bar{a}_{1}^{N} d_{2}^{N}}{c_{2}^{N} \bar{b}_{1}^{N}-a_{2}^{N} \bar{d}_{1}^{N}} \\
\bar{a}_{1}^{N} d_{4}^{N}=\bar{d}_{1}^{N} a_{2}^{N} \frac{b_{2}^{N} \bar{c}_{1}^{N}-\bar{a}_{1}^{N} d_{2}^{N}}{c_{2}^{N} \bar{b}_{1}^{N}-a_{2}^{N} \bar{d}_{1}^{N}} .
\end{array}
$$

Опять просто зачеркнем букву $N$, тогда

$$
\begin{aligned}
b_{1} \bar{b}_{1}=b_{3} \bar{b}_{3}, & b_{2} \bar{b}_{2}=b_{4} \bar{b}_{4}, \\
a_{1} \bar{a}_{1}=a_{3} \bar{a}_{3}, & a_{2} \bar{a}_{2}=a_{4} \bar{a}_{4}, \\
d_{1} \bar{b}_{1}=d_{3} \bar{b}_{3}, & a_{2} \bar{c}_{2}=a_{4} \bar{c}_{4}, \\
\frac{c_{2} d_{2}}{a_{2} b_{2}}=\frac{\bar{c}_{3} \bar{d}_{3}}{\bar{a}_{3} \bar{b}_{3}}, & \frac{c_{1} d_{1}}{a_{1} b_{1}}=\frac{c_{3} d_{3}}{a_{3} b_{3}}, \\
\frac{\bar{c}_{2} \bar{d}_{2}}{\bar{a}_{2} \bar{b}_{2}}=\frac{\bar{c}_{4} \bar{d}_{4}}{\bar{a}_{4} \bar{b}_{4}}, & \frac{c_{4} d_{4}}{a_{4} b_{4}}=\frac{\bar{c}_{1} \bar{d}_{1}}{\bar{a}_{1} \bar{b}_{1}} .
\end{aligned}
$$


Вспомним теперь про калибровочные симметрии $L$-оператора. При замене

$$
\begin{array}{rlrl}
a & \rightarrow \lambda a, & \bar{a} \rightarrow \lambda^{-1} \bar{a}, \\
c \rightarrow \lambda c, & \bar{c} \rightarrow \lambda^{-1} \bar{c}, \\
b \rightarrow b, & \bar{b} \rightarrow \bar{b}, \quad & d \rightarrow d, \quad \bar{d} \rightarrow \bar{d}
\end{array}
$$

оператор $L(u, p, \bar{p})$ не меняется. То же верно и для замены

$$
\begin{aligned}
b \rightarrow \lambda b, & \bar{b} \rightarrow \lambda^{-1} \bar{b} \\
d \rightarrow \lambda d, & \bar{d} \rightarrow \lambda^{-1} \bar{d}, \\
a \rightarrow a, \quad \bar{a} \rightarrow \bar{a}, & c \rightarrow c, \quad \bar{c} \rightarrow \bar{c} .
\end{aligned}
$$

Используя калибровочные степени свободы, можно положить

$$
b_{1}=b_{3}, \quad b_{2}=b_{4}, \quad a_{1}=a_{3}, \quad a_{2}=a_{4} .
$$

Отсюда и из соотношений (32) следует, что

$$
\begin{array}{llll}
\bar{b}_{1}=\bar{b}_{3}, & \bar{b}_{2}=\bar{b}_{4}, & \bar{a}_{1}=\bar{a}_{3}, & \bar{a}_{2}=\bar{a}_{4}, \\
d_{1}=d_{3}, & \bar{d}_{2}=\bar{d}_{4}, & c_{1}=c_{3}, & \bar{c}_{2}=\bar{c}_{4} .
\end{array}
$$

Кроме того, имеют место равенства

$$
\bar{c}_{3} \bar{d}_{3}=c_{2} d_{2} \frac{\bar{a}_{3} \bar{b}_{3}}{a_{2} b_{2}}, \quad c_{4} d_{4}=\bar{c}_{1} \bar{d}_{1} \frac{a_{2} b_{2}}{\bar{a}_{1} \bar{b}_{1}} .
$$

Вспоминая формулы (31), получим

$$
\begin{aligned}
& \bar{c}_{3}^{N}=\frac{c_{2}^{N} \bar{b}_{1}^{N}}{b_{2}^{N}} \frac{b_{2}^{N} \bar{c}_{1}^{N}-\bar{a}_{1}^{N} d_{2}^{N}}{c_{2}^{N} \bar{b}_{1}^{N}-a_{2}^{N} \bar{d}_{1}^{N}}, \\
& d_{4}^{N}=\frac{\bar{d}_{1}^{N} a_{2}^{N}}{\bar{a}_{1}^{N}} \frac{b_{2}^{N} \bar{c}_{1}^{N}-\bar{a}_{1}^{N} d_{2}^{N}}{c_{2}^{N} \bar{b}_{1}^{N}-a_{2}^{N} \bar{d}_{1}^{N}} .
\end{aligned}
$$

Найдем теперь матрицу $F$, сплетаюшую рассматриваемые нами два представления алгебры $L$-операторов. В частности, покажем, что условия (33)-(37) являются не только необходимыми, но и достаточными для существования $F$.

Итак, пусть

$$
F L_{1}\left(p_{1}, \bar{p}_{1}\right) L_{2}\left(p_{2}, \bar{p}_{2}\right)=L_{1}\left(p_{3}, \bar{p}_{3}\right) L_{2}\left(p_{4}, \bar{p}_{4}\right) F .
$$

Будем искать оператор $F$ в виде $F\left(X_{1} X_{2}^{-1}\right)$, где $X_{1}, X_{2}$-повышаюшие матрицы, действуюшие в первом и во втором $N$-мерном пространствах, соответственно. 
Как показано в приложении $\Gamma$, матрица $F$ действительно сушествует и выражается через $p_{1}, \bar{p}_{1}, p_{2}, \bar{p}_{2}$ при помоши формулы

$$
\frac{F\left(\bar{p}_{1}, p_{2} ; \omega^{k}\right)}{F\left(\bar{p}_{1}, p_{2} ; 1\right)}=\Omega\left(\bar{p}_{1}, p_{2}\right)^{-k} \prod_{j=1}^{k} \frac{\bar{c}_{1} b_{2}-\bar{a}_{1} d_{2} \omega^{j}}{\bar{b}_{1} c_{2}-\bar{d}_{1} a_{2} \omega^{j}}
$$

где для удобства введена функция

$$
\Omega\left(p_{1}, p_{2}\right)=\sqrt[N]{\frac{b_{1}^{N} c_{2}^{N}-d_{1}^{N} a_{2}^{N}}{c_{1}^{N} b_{2}^{N}-a_{1}^{N} d_{2}^{N}}}
$$

Подчеркнем, что $F$ зависит только от параметров $\bar{p}_{1}$ и $p_{2}$. При этом действие $F$ выражается формулами

$$
\begin{aligned}
& a_{3}=a_{1}, \quad \bar{a}_{3}=\bar{a}_{1}, \quad a_{4}=a_{2}, \quad \bar{a}_{4}=\bar{a}_{2}, \\
& b_{3}=b_{1}, \quad \bar{b}_{3}=\bar{b}_{1}, \quad b_{4}=b_{2}, \quad \bar{b}_{4}=\bar{b}_{2}, \\
& c_{3}=c_{1}, \quad d_{3}=d_{1}, \quad \bar{c}_{4}=\bar{c}_{2}, \quad \bar{d}_{4}=\bar{d}_{2}, \\
& \bar{c}_{3}=\Omega\left(\bar{p}_{1}, p_{2}\right) \frac{c_{2} \bar{b}_{1}}{b_{2}}, \quad c_{4}=\Omega\left(\bar{p}_{1}, p_{2}\right)^{-1} \frac{\bar{c}_{1} b_{2}}{\bar{b}_{1}}, \\
& \bar{d}_{3}=\Omega\left(\bar{p}_{1}, p_{2}\right)^{-1} \frac{d_{2} \bar{a}_{1}}{a_{2}}, \quad d_{4}=\Omega\left(\bar{p}_{1}, p_{2}\right) \frac{\bar{d}_{1} a_{2}}{\bar{a}_{1}} .
\end{aligned}
$$

Очевидно, что полученное выражение для $F$ является калибровочно-инвариантным.

Кроме указанных выше калибровочных степеней свободы, в случае произведения двух $L$-операторов имеется еше одна симметрия. А именно, между этими операторами можно вставить единицу, т.е.

$$
L_{1}\left(p_{3}, \bar{p}_{3}\right) L_{2}\left(p_{4}, \bar{p}_{4}\right)=L_{1}\left(p_{3}, \bar{p}_{3}\right) M^{-1} M L_{2}\left(p_{4}, \bar{p}_{4}\right),
$$

где $M-$ произвольная $(2 \times 2)$-матрица.

Пусть

$$
M=\left(\begin{array}{cc}
\bar{b}_{1} & 0 \\
b_{2} & \frac{\bar{a}_{1}}{a_{2}}
\end{array}\right) .
$$

Применим означенную симметрию к нашим $L$-операторам. В результате получим произведение новых $L$-операторов, параметры которых связаны с $p_{1}, \bar{p}_{1}, p_{2}, \bar{p}_{2}$ следующим образом:

$$
\begin{aligned}
& a_{3}=a_{1}, \quad \bar{a}_{3}=a_{2}, \quad a_{4}=\bar{a}_{1}, \quad \bar{a}_{4}=\bar{a}_{2}, \\
& b_{3}=b_{1}, \quad \bar{b}_{3}=b_{2}, \quad b_{4}=\bar{b}_{1}, \quad \bar{b}_{4}=\bar{b}_{2}, \\
& c_{3}=c_{1}, \quad d_{3}=d_{1}, \quad \bar{c}_{4}=\bar{c}_{2}, \quad \bar{d}_{4}=\bar{d}_{2}, \\
& \bar{c}_{3}=\Omega\left(\bar{p}_{1}, p_{2}\right) c_{2}, \quad c_{4}=\Omega\left(\bar{p}_{1}, p_{2}\right)^{-1} \bar{c}_{1}, \\
& \bar{d}_{3}=\Omega\left(\bar{p}_{1}, p_{2}\right)^{-1} d_{2}, \quad d_{4}=\Omega\left(\bar{p}_{1}, p_{2}\right) \bar{d}_{1} .
\end{aligned}
$$


Выражение для $F\left(\omega^{k}\right)$, конечно, при этом остается неизменным.

Случай, исследованный в работе [6], является частным случаем рассмотренной выше схемы при

$$
p_{3}=p_{1}, \quad \bar{p}_{3}=p_{2}, \quad p_{4}=\bar{p}_{1}, \quad \bar{p}_{4}=\bar{p}_{2} .
$$

В частности, нетрудно получить $F$-оператор, фигурируюший в соотношении (7). При этом на параметры $\bar{p}_{1}, p_{2}$ налагается дополнительное ограничение

$$
\Omega\left(\bar{p}_{1}, p_{2}\right)=1
$$

Полученные операторы $G$ и $F$ удовлетворяют соотношению, обобшающему соотношение "звезда-треугольник" для киральной модели Поттса [2]. Именно, имеет место соотношение

$$
G\left(\tilde{q}, \tilde{r} ; Z_{1}\right) F\left(\tilde{p}, r ; X_{1} X_{2}^{-1}\right) G\left(p, q ; Z_{1}\right)=\mu F\left(p^{\prime}, q^{\prime} ; X_{1} X_{2}^{-1}\right) G\left(p, r^{\prime} ; Z_{1}\right) F\left(q, r ; X_{1} X_{2}^{-1}\right)
$$

где $\mu$ - константа, а параметры, от которых зависят сплетаюшие операторы, связаны с $p, q, r$ при помощи следуюших формул:

$$
\begin{aligned}
& \begin{array}{l} 
\begin{cases}a_{r}^{\prime}=a_{r}, \\
b_{r}^{\prime}=b_{r}, \\
c_{r}^{\prime}=\Omega(q, r) c_{r}, \\
d_{r}^{\prime}=\Omega(q, r)^{-1} d_{r},\end{cases} \\
\left\{\begin{array}{l}
a_{p}^{\prime}=a_{p}, \\
b_{p}^{\prime}=b_{p}, \\
c_{p}^{\prime}=\Lambda\left(p, r^{\prime}\right)^{-1} c_{p}, \\
d_{p}^{\prime}=\Lambda\left(p, r^{\prime}\right) d_{p},
\end{array}\right. \\
\left\{\begin{array}{l}
\tilde{a}_{p}=a_{p}, \\
\tilde{b}_{p}=b_{p}, \\
\tilde{c}_{p}=\Lambda(p, q)^{-1} c_{p}, \\
\tilde{d}_{p}=\Lambda(p, q) d_{p},
\end{array}\right.
\end{array} \quad\left\{\begin{array}{l}
a_{q}^{\prime}=a_{q}, \\
d_{q}^{\prime}=\Omega(q, r)^{-1} c_{q},
\end{array}\right. \\
&
\end{aligned}
$$

Доказательство этого утверждения приводится в приложении Д.

ЗАмечАниЕ. Вьшеизложенное дает основание говорить о сушествовании новой алгебраической структуры, связанной с циклическими представлениями алгебры матриц монодромии.

Рассмотрим алгебру Хопфа с образуюшими $L_{i}^{j}\left(p_{1}, p_{2}\right), i, j=0,1, p_{1}, p_{2} \in \mathbb{C}^{4}$. Копроизведение задается формулой

$$
\Delta\left(L_{i}^{j}\right)=\left(L_{i}^{k}\right)_{1}\left(L_{k}^{j}\right)_{2} .
$$


Соотношения в алгебре Хопфа имеют вид

$$
\begin{gathered}
G\left(p_{1}, p_{2}\right) L_{i}^{j}\left(p_{1}, p_{2}\right)=L_{i}^{j}\left(\tilde{p}_{1}, \tilde{p}_{2}\right) G\left(p_{1}, p_{2}\right), \\
F\left(\bar{p}_{1}, p_{2}\right)\left(L_{i}^{k}\left(p_{1}, \bar{p}_{1}\right)\right)_{1}\left(L_{k}^{j}\left(p_{2}, \bar{p}_{2}\right)\right)_{2}=\left(L_{i}^{k}\left(p_{1}, \tilde{p}_{1}\right)\right)_{1}\left(L_{k}^{j}\left(\tilde{p}_{2}, \bar{p}_{2}\right)\right)_{2} F\left(\bar{p}_{1}, p_{2}\right), \\
\left(L_{i}^{k}\left(p_{1}, \bar{p}_{1}\right)\right)_{1}\left(L_{k}^{j}\left(p_{2}, \bar{p}_{2}\right)\right)_{2}=\left(L_{i}^{k}\left(p_{1}, \bar{p}_{1}\right)\right)_{1} M_{k}^{l}\left(M^{-1}\right)_{l}^{m}\left(L_{m}^{j}\left(p_{2}, \bar{p}_{2}\right)\right)_{2},
\end{gathered}
$$

где $M$ - произвольная диагональная двумерная матрица, а параметры в правых частях соотношений выражаются через параметры в левых частях при помоши формул (28), (40). По повторяюшимся индексам подразумевается суммирование.

\section{7. АЛГЕБРА $Q$-ОПЕРАТОРОВ}

Наряду с введенным в разделе 4 оператором $\mathcal{Q}(u)$, связанным с циклическими представлениями алгебры матриц монодромии в форме Бажанова-Строганова, можно рассматривать также оператор

$$
Q(u)=\operatorname{tr}_{0} L_{10}(u) L_{20}(u) \ldots L_{k 0}(u),
$$

где след берется по $N$-мерному пространству, а $L_{i 0}(u)$ - циклические представления алгебры матриц монодромии в форме Тарасова.

Операторы $Q(u)$ образуют алгебру, соотношения в которой следуют из свойств операторов $L(u)$ :

$$
\begin{gathered}
Q\left(\lambda p_{1}, \bar{p}_{1}\right)=\lambda^{k} Q\left(p_{1}, \bar{p}_{1}\right) \\
Q\left(p_{1}, \mu \bar{p}_{1}\right)=\mu^{k} Q\left(p_{1}, \bar{p}_{1}\right) \\
Q\left(a_{1}, b_{1}, c_{1}, d_{1}, \bar{a}_{1}, \bar{b}_{1}, \bar{c}_{1}, \bar{d}_{1}\right)=Q\left(\lambda a_{1}, b_{1}, \lambda c_{1}, d_{1}, \lambda^{-1} \bar{a}_{1}, \bar{b}_{1}, \lambda^{-1} \bar{c}_{1}, \bar{d}_{1}\right) \\
Q\left(a_{1}, b_{1}, c_{1}, d_{1}, \bar{a}_{1}, \bar{b}_{1}, \bar{c}_{1}, \bar{d}_{1}\right)=Q\left(a_{1}, \mu b_{1}, c_{1}, \mu d_{1}, \bar{a}_{1}, \mu^{-1} \bar{b}_{1}, \bar{c}_{1}, \mu^{-1} \bar{d}_{1}\right) \\
Q\left(a_{1}, b_{1}, c_{1}, d_{1}, \bar{a}_{1}, \bar{b}_{1}, \bar{c}_{1}, \bar{d}_{1}\right)=Q\left(\bar{a}_{1}, \bar{b}_{1}, \Lambda \bar{c}_{1}, \Lambda^{-1} \bar{d}_{1}, a_{1}, b_{1}, \Lambda^{-1} c_{1}, \Lambda d_{1}\right) \\
Q\left(a_{1}, b_{1}, c_{1}, d_{1}, \bar{a}_{1}, \bar{b}_{1}, \bar{c}_{1}, \bar{d}_{1}\right) Q\left(a_{2}, b_{2}, c_{2}, d_{2}, \bar{a}_{2}, \bar{b}_{2}, \bar{c}_{2}, \bar{d}_{2}\right)= \\
=Q\left(a_{1}, b_{1}, c_{1}, d_{1}, \beta \bar{a}_{1}, \alpha \bar{b}_{1}, \alpha \bar{c}_{1}, \beta \bar{d}_{1}\right) Q\left(\beta^{-1} a_{2}, \alpha^{-1} b_{2}, \alpha^{-1} c_{2}, \beta^{-1} d_{2}, \bar{a}_{2}, \bar{b}_{2}, \bar{c}_{2}, \bar{d}_{2}\right), \\
Q\left(a_{1}, b_{1}, c_{1}, d_{1}, \bar{a}_{1}, \bar{b}_{1}, \bar{c}_{1}, \bar{d}_{1}\right) Q\left(a_{2}, b_{2}, c_{2}, d_{2}, \bar{a}_{2}, \bar{b}_{2}, \bar{c}_{2}, \bar{d}_{2}\right)= \\
=Q\left(a_{1}, b_{1}, c_{1}, d_{1}, a_{2}, b_{2}, \Omega c_{2}, \Omega^{-1} d_{2}\right) Q\left(\bar{a}_{1}, \bar{b}_{1}, \Omega^{-1} \bar{c}_{1}, \Omega \bar{d}_{1}, \bar{a}_{2}, \bar{b}_{2}, \bar{c}_{2}, \bar{d}_{2}\right)
\end{gathered}
$$

где $\alpha, \beta, \lambda, \mu$-произвольные числа, $\Lambda=\Lambda\left(p_{1}, \bar{p}_{1}\right), \Omega=\Omega\left(\bar{p}_{1}, p_{2}\right)$.

Вывод всех этих соотношений приведен в приложении Е. 


\section{8. ЗАКЛЮЧЕНИЕ}

Отметим несколько вопросов, которые могут стать темой будуших исследований.

Подход, использованный в этой работе, может быть применен в более обших случаях. А именно, было бы интересно исследовать элементарные изоморфизмы, сплетаюшие циклические представления алгебры матриц монодромии, связанной с эллиптической $R$-матрицей, а также с $R$-матрицей, отвечающей квантовой алгебре $U_{\mathrm{q}}\left(s l_{n}\right)$.

Спектр трансфер-матрицы шестивершинной модели в корнях из единицы является вырож денным [5], [14]. Неодномерным собственным подпространствам трансфер-матрицы соответствуют некоторые конечномерные представления алгебры $Q$-операторов, которые действуют на этих пространствах нетривиальным образом, поскольку $Q$-операторы, вообше говоря, не коммутируют между собой.

В связи с этим исследование конечномерных представлений алгебры $Q$-операторов, задаваемой соотношениями (42)-(48), может пролить свет на свойства спектра оператора $T(u)$.

Также интересно было бы выяснить связь между алгеброй $Q$-операторов и симметрией $U\left(A_{1}^{1}\right)$, обнаруженной в работе $[14]^{1)}$.

\section{ПРИЛОЖЕНИЕ А}

\section{$T Q$-уравнение}

Найдем ядро оператора $\mathcal{L}_{23}\left(v ; p_{1}(v), p_{2}\right)=\tilde{\mathcal{L}}_{23}(v)$, где

$$
p_{1}(v)=\left(a_{1} v^{-1}, b_{1}, c_{1}, d_{1} v\right), \quad p_{2}=\left(a_{2}, b_{2}, c_{2}, d_{2}\right) .
$$

Имеем

$$
\tilde{\mathcal{L}}_{23}(v)=\left(\begin{array}{cc}
v^{-1} c_{1} c_{2} Z-b_{1} b_{2} v Z^{-1} & -v\left(b_{1} d_{2} Z^{-1}-c_{1} a_{2} Z\right) X \\
\omega v^{-1} X^{-1}\left(d_{1} b_{2} v Z^{-1}-a_{1} c_{2} v^{-1} Z\right) & d_{1} d_{2} Z^{-1}-a_{1} a_{2} \omega^{2} Z
\end{array}\right) .
$$

Выберем базис $|\alpha\rangle, \alpha=0, \ldots, N-1(\bmod N)$ :

$$
Z|\alpha\rangle=\omega^{\alpha}|\alpha\rangle, \quad X|\alpha\rangle=|\alpha+1\rangle .
$$

Рассмотрим $2 N$-мерньй вектор

$$
\Psi=\left(\begin{array}{c}
\Phi_{1} \\
\Phi_{2}
\end{array}\right)
$$

где $\Phi_{1}, \Phi_{2}-N$-мерные векторы. Действуя на вектор $\Psi$ оператором $\tilde{\mathcal{L}}_{23}(v)$ и приравнивая результат нулю, получим систему уравнений

$$
\begin{aligned}
\left(c_{1} c_{2} Z-b_{1} b_{2} v^{2} Z^{-1}\right) \Phi_{1}-v^{2}\left(b_{1} d_{2} Z^{-1}-c_{1} a_{2} Z\right) X \Phi_{2}=0 \\
\omega X^{-1} v^{-1}\left(d_{1} b_{2} v Z^{-1}-a_{1} c_{2} v^{-1} Z\right) \Phi_{1}+\left(d_{1} d_{2} Z^{-1}-a_{1} a_{2} \omega^{2} Z\right) \Phi_{2}=0 .
\end{aligned}
$$

\footnotetext{
1) После направления статьи в печать мы узнали, что изоморфизмы между представлениями алгебры монодромии в корнях из единицы изучались Пакуляком и Сергеевым для случая релятивистской цепочки Тоды [15].
} 
Нетрудно увидеть, что эта система имеет решения тогда и только тогда, когда

$$
v^{2}=v_{*}^{2}=\frac{c_{2} d_{2}}{a_{2} b_{2}}
$$

Векторами, образуюшими ядро, при этом являются

$$
\Psi_{\alpha}=\left(\begin{array}{c}
-d_{2}|\alpha\rangle \\
b_{2}|\alpha-1\rangle
\end{array}\right)=-d_{2}|0, \alpha\rangle+b_{2}|1, \alpha-1\rangle
$$

Теперь мы должны подействовать на векторы $\Psi_{\alpha}$ оператором $\tilde{\mathcal{L}}_{13}\left(u v_{*}\right) \mathcal{R}_{12}(u)$ (особо следует обратить внимание на то, что здесь можно пользоваться обычным матричным умножением по двумерному пространству).

Пусть

$$
\tilde{\mathcal{L}}_{13}\left(u v_{*}\right)=\left(\begin{array}{ll}
A\left(u v_{*}\right) & B\left(u v_{*}\right) \\
C\left(u v_{*}\right) & D\left(u v_{*}\right)
\end{array}\right), \quad \mathcal{R}_{12}(u)=\left(\begin{array}{cc}
a(u) & b(u) \\
c(u) & d(u)
\end{array}\right) .
$$

Тогда

$$
\tilde{\mathcal{L}}_{13}\left(u v_{*}\right) \mathcal{R}_{12}(u)=\left(\begin{array}{cc}
a(u) A\left(u v_{*}\right)+c(u) B\left(u v_{*}\right) & b(u) A\left(u v_{*}\right)+d(u) B\left(u v_{*}\right) \\
a(u) C\left(u v_{*}\right)+c(u) D\left(u v_{*}\right) & b(u) C\left(u v_{*}\right)+d(u) D\left(u v_{*}\right)
\end{array}\right) .
$$

Действуя каждым из четырех матричных элементов на вектор $\Psi_{\alpha}$, получим, например,

$$
\begin{aligned}
(\tilde{\mathcal{L}} \mathcal{R})_{0}^{0} \Psi_{\alpha}= & {\left[a(u) A\left(u v_{*}\right)+c(u) B\left(u v_{*}\right)\right]\left[-d_{2}|0, \alpha\rangle+b_{2}|1, \alpha-1\rangle\right]=} \\
= & -d_{2}\left(u \omega-u^{-1} \omega^{-1}\right)\left(v_{*}^{-1} u^{-1} c_{1} c_{2} \omega^{\alpha}-b_{1} b_{2} u v_{*} \omega^{-\alpha}\right)|0, \alpha\rangle+ \\
& +\omega b_{2}\left\{\left(u-u^{-1}\right)\left(v_{*}^{-1} u^{-1} c_{1} c_{2} \omega^{\alpha-1}-b_{1} b_{2} u v_{*} \omega^{1-\alpha}\right)|1, \alpha-1\rangle+\right. \\
& \left.+u\left(\omega-\omega^{-1}\right)\left(-u v_{*}\right)\left(b_{1} d_{2} \omega^{-\alpha}-c_{1} a_{2} \omega^{\alpha}\right)|0, \alpha\rangle\right\}= \\
= & \left(u-u^{-1}\right)\left(v_{*}^{-1} u^{-1} c_{1} c_{2} \omega^{\alpha-1}-b_{1} b_{2} v_{*} u \omega^{1-\alpha}\right) \Psi_{\alpha} .
\end{aligned}
$$

Аналогично

$$
\begin{aligned}
& (\tilde{\mathcal{L}} \mathcal{R})_{1}^{1} \Psi_{\alpha}=\left(u-u^{-1}\right)\left(d_{1} d_{2} \omega^{-\alpha}-a_{1} a_{2} \omega^{2+\alpha}\right) \Psi_{\alpha} \\
& (\tilde{\mathcal{L}} \mathcal{R})_{0}^{1} \Psi_{\alpha}=\left(u-u^{-1}\right)\left(-v_{*} u\right)\left(b_{1} d_{2} \omega^{-\alpha-1}-c_{1} a_{2} \omega^{\alpha+1}\right) \Psi_{\alpha+1} \\
& (\tilde{\mathcal{L}} \mathcal{R})_{1}^{0} \Psi_{\alpha}=\left(u-u^{-1}\right) \omega v_{*}^{-1} u^{-1}\left(d_{1} b_{2} u v_{*} \omega^{1-\alpha}-a_{1} c_{2} u^{-1} v_{*}^{-1} \omega^{\alpha-1}\right) \Psi_{\alpha-1}
\end{aligned}
$$

Очевидно, что ядро $\tilde{\mathcal{L}}_{23}\left(v_{*}\right)$ действительно является инвариантным подпространством относительно оператора $\tilde{\mathcal{L}}_{13}\left(u v_{*}\right) \mathcal{R}_{12}(u)$.

Нетрудно увидеть, что полученная $N$-мерная матрица пропорциональна $\tilde{\mathcal{L}}\left(u v_{*} \omega\right)$, т.е.

$$
\left.\tilde{\mathcal{L}}_{13}\left(u v_{*}\right) \mathcal{R}_{12}(u)\right|_{\operatorname{Ker} \mathcal{L}_{23}}=\left(u-u^{-1}\right) \tilde{\mathcal{L}}\left(u v_{*} \omega\right) .
$$


Рассмотрим теперь векторы, принадлежащие дополнению ядра. Если провести факторизацию по векторам самого ядра, то это дополнение также должно быть инвариантным пространством относительно оператора $\tilde{\mathcal{L}}_{13}\left(u v_{*}\right) \mathcal{R}_{12}(u)$. В качестве базиса выберем векторы $\Psi_{\alpha}^{\prime}=|0, \alpha\rangle$. Проведя вычисления, получим $\left(\right.$ по $\left.\bmod \Psi_{\alpha}\right)$ :

$$
\begin{aligned}
& (\tilde{\mathcal{L}} \mathcal{R})_{0}^{0} \Psi_{\alpha}^{\prime}=\left(u \omega-u^{-1} \omega^{-1}\right)\left(v_{*}^{-1} u^{-1} c_{1} c_{2} \omega^{\alpha}-b_{1} b_{2} v_{*} u \omega^{-\alpha}\right) \Psi_{\alpha}^{\prime} \\
& (\tilde{\mathcal{L}} \mathcal{R})_{1}^{1} \Psi_{\alpha}^{\prime}=\left(u \omega-u^{-1} \omega^{-1}\right)\left(d_{1} d_{2} \omega^{1-\alpha}-a_{1} a_{2} \omega^{1+\alpha}\right) \Psi_{\alpha}^{\prime} \\
& (\tilde{\mathcal{L}} \mathcal{R})_{0}^{1} \Psi_{\alpha}^{\prime}=\left(u \omega-u^{-1} \omega^{-1}\right)\left(-v_{*} u\right)\left(b_{1} d_{2} \omega^{-\alpha}-c_{1} a_{2} \omega^{\alpha}\right) \Psi_{\alpha+1}^{\prime} \\
& (\tilde{\mathcal{L}} \mathcal{R})_{1}^{0} \Psi_{\alpha}^{\prime}=\left(u \omega-u^{-1} \omega^{-1}\right) \omega v_{*}^{-1} u^{-1}\left(d_{1} b_{2} u v_{*} \omega^{-\alpha}-a_{1} c_{2} u^{-1} v_{*}^{-1} \omega^{\alpha}\right) \Psi_{\alpha-1}^{\prime}
\end{aligned}
$$

Как и в предыдушем случае, полученная $N$-мерная матрица пропорциональна $\tilde{\mathcal{L}}\left(u v_{*} \omega^{-1}\right)$, т.е.

$$
\left.\tilde{\mathcal{L}}_{13}\left(u v_{*}\right) \mathcal{R}_{12}(u)\right|_{\left(\operatorname{Ker} \mathcal{L}_{23}\right)^{\perp}}=\left(u \omega-u^{-1} \omega^{-1}\right) \tilde{\mathcal{L}}\left(u v_{*} \omega^{-1}\right) .
$$

Таким образом, мы получили, что условия (13) действительно выполняются. При этом

$$
\lambda=\omega, \quad \phi_{1}=u-u^{-1}, \quad \phi_{2}=u \omega-u^{-1} \omega^{-1}
$$

и имеет место $T Q$-уравнение

$$
\mathcal{Q}(u) T(u)=\left(u-u^{-1}\right)^{n} \mathcal{Q}(u \omega)+\left(u \omega-u^{-1} \omega^{-1}\right)^{n} \mathcal{Q}\left(u \omega^{-1}\right),
$$

где

$$
\mathcal{Q}(u)=\operatorname{tr}_{3} \tilde{\mathcal{L}}_{13}(u) \tilde{\mathcal{L}}_{1^{\prime} 3}(u) \ldots \tilde{\mathcal{L}}_{1^{(n)} 3}(u) .
$$

ПРИЛОЖЕНИЕ Б

\section{Условия эквивалентности представлений. Кривая Ферма}

Пусть $N$ - нечетное число. Докажем, что представления $L_{1}\left(u, p_{1}, p_{2}\right) L_{2}\left(u, p_{3}, p_{4}\right)$ и $L_{1}\left(u, p_{3}, p_{4}\right) L_{2}\left(u, p_{1}, p_{2}\right)$ в обшем случае эквивалентны тогда и только тогда, когда можно выбрать $p_{i}(i=1,2,3,4)$, удовлетворяющие условиям

$$
\frac{a_{i}^{N} \pm b_{i}^{N}}{c_{i}^{N} \pm d_{i}^{N}}=\lambda_{ \pm}
$$

определяюшим кривую Ферма.

Для удобства везде в дальнейшем произведем следуюшие переобозначения:

$$
a_{i}^{N} \rightarrow a_{i}, \quad b_{i}^{N} \rightarrow b_{i}, \quad c_{i}^{N} \rightarrow c_{i}, \quad d_{i}^{N} \rightarrow d_{i}
$$

Имеем

$$
\begin{aligned}
\left\langle L\left(u, p_{1}, p_{2}\right)\right\rangle & =\left(\begin{array}{cc}
c_{1} c_{2}-b_{1} b_{2} u & -u\left(b_{1} d_{2}-c_{1} a_{2}\right) \\
d_{1} b_{2}-a_{1} c_{2} & d_{1} d_{2}-a_{1} a_{2} u
\end{array}\right), \\
\left\langle L\left(u, p_{3}, p_{4}\right)\right\rangle & =\left(\begin{array}{cc}
c_{3} c_{4}-b_{3} b_{4} u & -u\left(b_{3} d_{4}-c_{3} a_{4}\right) \\
d_{3} b_{4}-a_{3} c_{4} & d_{3} d_{4}-a_{3} a_{4} u
\end{array}\right) .
\end{aligned}
$$


Два представления $L_{\pi}, L_{\pi^{\prime}}$ будут эквивалентными, если существует изоморфизм $P$ такой, что

$$
L_{\pi^{\prime}}=P L_{\pi} P^{-1}
$$

т.е. имеется взаимно однозначное соответствие между элементами в $L_{\pi}$ и $L_{\pi^{\prime}}$. Центральные элементы $\left\langle L_{\pi}\right\rangle$ и $\left\langle L_{\pi^{\prime}}\right\rangle$ при этом должны быть одинаковы [6]. Если $\pi=\pi_{1} \times \pi_{2}$, $\pi^{\prime}=\pi_{2} \times \pi_{1}$, то

$$
\left\langle L_{\pi_{1}}\right\rangle\left\langle L_{\pi_{2}}\right\rangle=\left\langle L_{\pi_{2}}\right\rangle\left\langle L_{\pi_{1}}\right\rangle
$$

Отсюда следует, что

$$
\left\langle L\left(u, p_{1}, p_{2}\right)\right\rangle\left\langle L\left(u, p_{3}, p_{4}\right)\right\rangle=\left\langle L\left(u, p_{3}, p_{4}\right)\right\rangle\left\langle L\left(u, p_{1}, p_{2}\right)\right\rangle .
$$

Перемножая матрицы, получаем пять уравнений, из которых только три являются независимыми:

$$
\begin{aligned}
& \frac{b_{1} d_{2}-c_{1} a_{2}}{d_{1} b_{2}-a_{1} c_{2}}=\frac{b_{3} d_{4}-c_{3} a_{4}}{d_{3} b_{4}-a_{3} c_{4}}=s, \\
& \frac{a_{1} a_{2}-b_{1} b_{2}}{b_{1} d_{2}-c_{1} a_{2}}=\frac{a_{3} a_{4}-b_{3} b_{4}}{b_{3} d_{4}-c_{3} a_{4}}=q, \\
& \frac{c_{1} c_{2}-d_{1} d_{2}}{b_{1} d_{2}-c_{1} a_{2}}=\frac{c_{3} c_{4}-d_{3} d_{4}}{b_{3} d_{4}-c_{3} a_{4}}=r,
\end{aligned}
$$

где $s, q, r$-произвольные константы

Эта система уравнений имеет решения при следуюших ограничениях на $p_{i}$ :

$$
\begin{aligned}
& b_{1} d_{2}-c_{1} a_{2}=s\left(d_{1} b_{2}-a_{1} c_{2}\right), \\
& a_{1} a_{2}-b_{1} b_{2}=q\left(b_{1} d_{2}-c_{1} a_{2}\right), \\
& c_{1} c_{2}-d_{1} d_{2}=r\left(b_{1} d_{2}-c_{1} a_{2}\right) .
\end{aligned}
$$

Оказьвается, что (Б.2) может выполняться, если $p_{1}$ и $p_{2}$ - точки кривой, полученной пересечением двух плоскостей (проективная симметрия оператора $L$ ), т.е.

$$
\begin{aligned}
& \alpha_{1} a_{i}+\beta_{1} b_{i}+\gamma_{1} c_{i}+\delta_{1} d_{i}=0, \\
& \alpha_{2} a_{i}+\beta_{2} b_{i}+\gamma_{2} c_{i}+\delta_{2} d_{i}=0, \quad i=1,2 .
\end{aligned}
$$

Найдем эти многообразия. Из последней системы имеем

$$
\begin{aligned}
a_{i} & =\lambda_{i} c_{i}+\mu_{i} d_{i}, \\
b_{i} & =\nu_{i} c_{i}+\eta_{i} d_{i}, \quad i=1,2 .
\end{aligned}
$$

Подставляем (Б.3) в (Б.2). Получаем систему уравнений для коэффициентов

$$
\begin{gathered}
\eta_{1}=s \eta_{2}, \quad \lambda_{2}=s \lambda_{1}, \quad \nu_{1}=\mu_{2}, \quad \nu_{2}=\mu_{1}, \\
1=-r \lambda_{2}, \quad-1=r \eta_{1}, \quad \nu_{1}=\mu_{2}, \\
\lambda_{1} \lambda_{2}-\nu_{1} \nu_{2}=-q \lambda_{2}, \quad \mu_{1} \mu_{2}-\eta_{1} \eta_{2}=q \eta_{1}, \\
\lambda_{1} \mu_{2}-\nu_{1} \eta_{2}=q \nu_{1}-q \mu_{2}, \quad \mu_{1} \lambda_{2}-\nu_{2} \eta_{1}=0 .
\end{gathered}
$$


Решая эту систему, получим

$$
\begin{gathered}
\nu_{1}=\mu_{2}=\mu, \quad \mu_{1}=\nu_{2}=\nu, \\
\eta_{1}=\lambda_{2}=\lambda, \quad \lambda_{1}=\eta_{2}=\eta, \\
\lambda=-\frac{1}{r}, \quad \nu \mu=(q+\eta) \lambda, \quad s=1 .
\end{gathered}
$$

Кроме того, поскольку точки $p_{1}$ и $p_{2}$ лежат на одной и той же кривой, то

$$
\lambda_{1}=\lambda_{2}=\lambda, \quad \mu_{1}=\mu_{2}=\mu, \quad \nu_{1}=\nu_{2}=\nu, \quad \eta_{1}=\eta_{2}=\eta
$$

Следовательно,

$$
\lambda=\eta, \quad \nu=\mu
$$

В итоге получаем, что

$$
\frac{a_{1}+b_{1}}{c_{1}+d_{1}}=\frac{a_{2}+b_{2}}{c_{2}+d_{2}}, \quad \frac{a_{1}-b_{1}}{c_{1}-d_{1}}=\frac{a_{2}-b_{2}}{c_{2}-d_{2}} .
$$

Аналогично можно установить, что

$$
\frac{a_{3}+b_{3}}{c_{3}+d_{3}}=\frac{a_{4}+b_{4}}{c_{4}+d_{4}}, \quad \frac{a_{3}-b_{3}}{c_{3}-d_{3}}=\frac{a_{4}-b_{4}}{c_{4}-d_{4}} .
$$

Вернемся к старым обозначениям

$$
p_{i} \rightarrow p_{i}^{N}, \quad i=1,2,3,4
$$

В силу (Б.1) в обшем случае точки $p_{1}, p_{2}, p_{3}, p_{4}$ могут быть связаны следуюшими двумя способами:
a) $\frac{a_{1}^{N} \pm b_{1}^{N}}{c_{1}^{N} \pm d_{1}^{N}}=\frac{a_{2}^{N} \pm b_{2}^{N}}{c_{2}^{N} \pm d_{2}^{N}}=\frac{a_{3}^{N} \pm b_{3}^{N}}{c_{3}^{N} \pm d_{3}^{N}}=\frac{a_{4}^{N} \pm b_{4}^{N}}{c_{4}^{N} \pm d_{4}^{N}}$
б) $\frac{a_{1}^{N} \pm b_{1}^{N}}{c_{1}^{N} \pm d_{1}^{N}}=\frac{a_{2}^{N} \pm b_{2}^{N}}{c_{2}^{N} \pm d_{2}^{N}}=\frac{a_{3}^{N} \mp b_{3}^{N}}{c_{3}^{N} \mp d_{3}^{N}}=\frac{a_{4}^{N} \mp b_{4}^{N}}{c_{4}^{N} \mp d_{4}^{N}}$.

Это обусловлено наличием двух корней уравнения

$$
\nu^{2}=\mu^{2}=-\frac{q}{r}+\frac{1}{r^{2}} .
$$

Однако следует вспомнить про симметрии оператора $L\left(u, p_{1}, p_{2}\right)$, когда можно сделать замену

$$
b_{1} \rightarrow \lambda b_{1}, \quad b_{2} \rightarrow \lambda^{-1} b_{2}, \quad d_{1} \rightarrow \lambda d_{1}, \quad d_{2} \rightarrow \lambda^{-1} d_{2}
$$

и $L\left(u, p_{1}, p_{2}\right)$ не изменится. Посредством подобной замены с $\lambda=-1$ случай “б” сводится к случаю "а" (напомним, что число $N$ нечетно). 
ПРИЛОЖЕНИЕ В

\section{Условия эквивалентности представлений в общем случае}

Введем обозначения

$$
\begin{array}{lll}
\phi=d^{N} \bar{b}^{N}, & \psi=a^{N} \bar{c}^{N}, & \beta=b^{N} \bar{b}^{N}, \\
\delta=a^{N} \bar{a}^{N}, & \mu=\frac{c^{N} d^{N}}{a^{N} b^{N}}, & \lambda=\frac{\bar{c}^{N} \bar{d}^{N}}{\bar{a}^{N} \bar{b}^{N}} .
\end{array}
$$

Тогда $\langle L(u, p, \bar{p})\rangle$ можно записать в виде

$$
\langle L(u, p, \bar{p})\rangle=\left(\begin{array}{cc}
\frac{\mu \beta \psi}{\phi}-\beta u & -u\left(\frac{\lambda \beta \delta}{\psi}-\frac{\mu \beta \delta}{\phi}\right) \\
\phi-\psi & \frac{\lambda \delta \phi}{\psi}-\delta u
\end{array}\right) .
$$

Рассмотрим два представления алгебры $L$-операторов $L\left(u, p_{1}, \bar{p}_{1}\right)$ и $L\left(u, p_{2}, \bar{p}_{2}\right)$ и найдем условия, при которых они эквивалентны. Необходимым условием эквивалентности двух представлений является совпадение центров этих представлений:

$$
\left\langle L\left(u, p_{1}, \bar{p}_{1}\right)\right\rangle=\left\langle L\left(u, p_{2}, \bar{p}_{2}\right)\right\rangle .
$$

Отсюда получаем, приравнивая коэффициенты при степенях $u$, что

$$
\begin{gathered}
\delta_{1}=\delta_{2}, \quad \beta_{1}=\beta_{2}, \quad \phi_{1}-\psi_{1}=\phi_{2}-\psi_{2} \\
\frac{\lambda_{1} \phi_{1}}{\psi_{1}}=\frac{\lambda_{2} \phi_{2}}{\psi_{2}}
\end{gathered}
$$

Кроме того, из (В.1) следует, что

$$
\operatorname{det}\left\langle L\left(u, p_{1}, \bar{p}_{1}\right)\right\rangle=\operatorname{det}\left\langle L\left(u, p_{2}, \bar{p}_{2}\right)\right\rangle .
$$

В последнем уравнении левая и правая части являются полиномами второй степени от $u$. Корни левого полинома суть

$$
u_{1}=\lambda_{1}, \quad \bar{u}_{1}=\mu_{1},
$$

а корни правого -

$$
u_{2}=\lambda_{2}, \quad \bar{u}_{2}=\mu_{2} .
$$

Ясно, что корни левой и правой частей должны совпадать. Будем рассматривать следуюший вариант:

$$
\lambda_{1}=\mu_{2}, \quad \lambda_{2}=\mu_{1} .
$$


Записывая (В.2)-(В.4) с помошью параметров $a_{i}, b_{i}, c_{i}, d_{i}$, получим

$$
\begin{gathered}
a_{1}^{N} \bar{a}_{1}^{N}=a_{2}^{N} \bar{a}_{2}^{N}, \quad b_{1}^{N} \bar{b}_{1}^{N}=b_{2}^{N} \bar{b}_{2}^{N}, \\
\frac{\bar{c}_{1}^{N} \bar{d}_{1}^{N}}{\bar{a}_{1}^{N} \bar{b}_{1}^{N}}=\frac{c_{2}^{N} d_{2}^{N}}{a_{2}^{N} b_{2}^{N}}, \quad \frac{\bar{c}_{2}^{N} \bar{d}_{2}^{N}}{\bar{a}_{2}^{N} \bar{b}_{2}^{N}}=\frac{c_{1}^{N} d_{1}^{N}}{a_{1}^{N} b_{1}^{N}}, \\
\frac{d_{1}^{N} \bar{d}_{1}^{N}}{a_{1}^{N} \bar{a}_{1}^{N}}=\frac{d_{2}^{N} \bar{d}_{2}^{N}}{a_{2}^{N} \bar{a}_{2}^{N}}, \\
d_{1}^{N} \bar{b}_{1}^{N}-a_{1}^{N} \bar{c}_{1}^{N}=d_{2}^{N} \bar{b}_{2}^{N}-a_{2}^{N} \bar{c}_{2}^{N} .
\end{gathered}
$$

Рассмотрим теперь два представления алгебры $L$-операторов $L_{1}\left(u, p_{1}, \bar{p}_{1}\right) \times$ $L_{2}\left(u, p_{2}, \bar{p}_{2}\right)$ и $L_{1}\left(u, p_{3}, \bar{p}_{3}\right) L_{2}\left(u, p_{4}, \bar{p}_{4}\right)$. Мы хотим найти условия, при которых эти два представления будут эквивалентными. Имеем

$$
\operatorname{det}\left\langle L_{1}\left(u, p_{1}, \bar{p}_{1}\right)\right\rangle \operatorname{det}\left\langle L_{2}\left(u, p_{2}, \bar{p}_{2}\right)\right\rangle=\operatorname{det}\left\langle L_{1}\left(u, p_{3}, \bar{p}_{3}\right)\right\rangle \operatorname{det}\left\langle L_{2}\left(u, p_{4}, \bar{p}_{4}\right)\right\rangle .
$$

Каждый детерминант является квадратным многочленом относительно $u$. Нетрудно найти его корни

$$
u=\lambda, \quad \bar{u}=\mu .
$$

В итоге мы видим, что левая часть (В.5) обрашается в нуль при $u_{1}=\lambda_{1}, \bar{u}_{1}=\mu_{1}, u_{2}=$ $\lambda_{2}, \bar{u}_{2}=\mu_{2}$, а правая - при $u_{3}=\lambda_{3}, \bar{u}_{3}=\mu_{3}, u_{4}=\lambda_{4}, \bar{u}_{4}=\mu_{4}$. Ясно, что должно быть совпадение корней слева и справа. Будем рассматривать следующий вариант:

$$
\mu_{2}=\lambda_{3}, \quad \mu_{1}=\mu_{3}, \quad \lambda_{2}=\lambda_{4}, \quad \lambda_{1}=\mu_{4} .
$$

Тогда

$$
\left\langle L_{1}\left(u, p_{1}, \bar{p}_{1}\right)\right\rangle\left\langle L_{2}\left(u, p_{2}, \bar{p}_{2}\right)\right\rangle=\left\langle L_{1}\left(u, p_{3}, \bar{p}_{3}\right)\right\rangle\left\langle L_{2}\left(u, p_{4}, \bar{p}_{4}\right)\right\rangle,
$$

Отдельным сомножителям в левой и правой частях уравнения (В.6) отвечают соответственно корни

$$
\mu_{1}, \lambda_{1} ; \quad \mu_{2}, \lambda_{2} ; \quad \mu_{1}, \mu_{2} ; \quad \lambda_{1}, \lambda_{2} .
$$

Мы видим, что два корня $\lambda_{1}$ и $\mu_{2}$ поменялись местами.

Пусть $u=\lambda_{2}$. Подействуем слева на вектор $\Psi_{1}$, который является правым нулевым вектором оператора $\left\langle L_{2}\left(\lambda_{2}, p_{2}, \bar{p}_{2}\right)\right\rangle$ :

$$
\Psi_{1}=\left(\begin{array}{c}
-\lambda_{2} \delta_{2} \\
\psi_{2}
\end{array}\right), \quad\left\langle L_{2}\left(\lambda_{2}, p_{2}, \bar{p}_{2}\right)\right\rangle \Psi_{1}=0
$$

обеими частями уравнения (В.6). Этот вектор является правым нулевым и для оператора $\left\langle L_{2}\left(\lambda_{2}, p_{4}, \bar{p}_{4}\right)\right\rangle$. Следовательно, имеет место равенство

$$
\frac{\delta_{2}}{\psi_{2}}=\frac{\delta_{4}}{\psi_{4}}
$$


Пусть теперь $u=\mu_{1}$. Подействуем, но теперь уже справа, обеими частями (В.6) на левый нулевой вектор $\Psi_{2}$ оператора $\left\langle L_{1}\left(\mu_{1}, p_{1}, \bar{p}_{1}\right)\right\rangle$ :

$$
\Psi_{2}=\left(\begin{array}{c}
\phi_{1} \\
\mu_{1} \beta_{1}
\end{array}\right), \quad \Psi_{2}\left\langle L_{1}\left(\mu_{1}, p_{1}, \bar{p}_{1}\right)\right\rangle=0 .
$$

Поскольку этот вектор является также левым нулевым вектором оператора $\left\langle L_{1}\left(\mu, p_{3}, \bar{p}_{3}\right)\right\rangle$, то

$$
\frac{\phi_{1}}{\beta_{1}}=\frac{\phi_{3}}{\beta_{3}} .
$$

Очевидно, (В.6) останется справедливым, если вставить между двумя множителями в правой части $1=M M^{-1}$, где $M-$ двумерная матрица

$$
M=\left(\begin{array}{cc}
m_{1} & 0 \\
0 & m_{2}
\end{array}\right) .
$$

Используя эту калибровочную симметрию, можно положить

$$
\beta_{3}=\beta_{1}, \quad \delta_{4}=\delta_{2},
$$

откуда сразу же следует, что

$$
\phi_{3}=\phi_{1}, \quad \psi_{4}=\psi_{2} .
$$

Непосредственно перемножая матрицы в (В.6) и приравнивая коэффициенты при степенях $u$, нетрудно получить

$$
\begin{gathered}
\beta_{4}=\beta_{2}, \quad \delta_{3}=\delta_{1}, \\
\psi_{3}=\phi_{4} \frac{\mu_{2} \psi_{1}}{\lambda_{1} \phi_{2}}, \\
\psi_{3} \beta_{2}-\delta_{1} \phi_{4}=\psi_{1} \beta_{2}-\delta_{1} \phi_{2} .
\end{gathered}
$$

Из последних двух равенств следует, что

$$
\phi_{4}=\frac{\lambda_{1} \phi_{2}\left(\beta_{2} \psi_{1}-\delta_{1} \phi_{2}\right)}{\beta_{2} \mu_{2} \psi_{1}-\lambda_{1} \delta_{1} \phi_{2}}, \quad \psi_{3}=\frac{\mu_{2} \psi_{1}\left(\beta_{2} \psi_{1}-\delta_{1} \phi_{2}\right)}{\beta_{2} \mu_{2} \psi_{1}-\lambda_{1} \delta_{1} \phi_{2}} .
$$

Собирая все полученные равенства, кроме (В.7), получим

$$
\begin{aligned}
\beta_{1}=\beta_{3}, \quad \beta_{2}=\beta_{4}, \quad \delta_{1}=\delta_{3}, \quad \delta_{2}=\delta_{4}, \\
\phi_{1}=\phi_{3}, \quad \psi_{2}=\psi_{4}, \quad \mu_{2}=\lambda_{3}, \\
\mu=\mu_{3}, \quad \lambda_{2}=\lambda_{4}, \quad \lambda_{1}=\mu_{4} .
\end{aligned}
$$




\section{Вычисление оператора $F$}

\section{ПРИЛОЖЕНИЕ Г}

Матричное уравнение (38) можно записать в виде системы уравнений, соответствуюших четырем матричным элементам. Имеем

$$
L_{1}\left(p_{1}, \bar{p}_{1}\right) L_{2}\left(p_{2}, \bar{p}_{2}\right)=\left(\begin{array}{cc}
A_{1} & B_{1} \\
C_{1} & D_{1}
\end{array}\right)\left(\begin{array}{ll}
A_{2} & B_{2} \\
C_{2} & D_{2}
\end{array}\right)=\left(\begin{array}{ll}
A_{1} A_{2}+B_{1} C_{2} & A_{1} B_{2}+B_{1} D_{2} \\
C_{1} A_{2}+D_{1} C_{2} & C_{1} B_{2}+D_{1} D_{2}
\end{array}\right) .
$$

В результате получаем систему уравнений

$$
\begin{aligned}
& F\left(X_{1} X_{2}^{-1}\right)\left(A_{1} A_{2}+B_{1} C_{2}\right)=\left(A_{3} a_{4}+B_{3} C_{4}\right) F\left(X_{1} X_{2}^{-1}\right), \\
& F\left(X_{1} X_{2}^{-1}\right)\left(A_{1} B_{2}+B_{1} D_{2}\right)=\left(A_{3} B_{4}+B_{3} D_{4}\right) F\left(X_{1} X_{2}^{-1}\right), \\
& F\left(X_{1} X_{2}^{-1}\right)\left(C_{1} A_{2}+D_{1} C_{2}\right)=\left[C_{3} a_{4}+D_{3} C_{4}\right] F\left(X_{1} X_{2}^{-1}\right), \\
& F\left(X_{1} X_{2}^{-1}\right)\left(C_{1} B_{2}+D_{1} D_{2}\right)=\left(C_{3} B_{4}+D_{3} D_{4}\right) F\left(X_{1} X_{2}^{-1}\right) .
\end{aligned}
$$

Выбираем базис $\left|k_{1}, k_{2}\right\rangle, k_{1}, k_{2}=0, \ldots, N-1(\bmod N)$,

$$
\begin{aligned}
X_{1}\left|k_{1}, k_{2}\right\rangle & =\omega^{k_{1}}\left|k_{1}, k_{2}\right\rangle, \quad X_{2}\left|k_{1}, k_{2}\right\rangle=\omega^{k_{2}}\left|k_{1}, k_{2}\right\rangle, \\
Z_{1}\left|k_{1}, k_{2}\right\rangle & =\left|k_{1}-1, k_{2}\right\rangle, \quad Z_{2}\left|k_{1}, k_{2}\right\rangle=\left|k_{1}, k_{2}-1\right\rangle .
\end{aligned}
$$

В этом базисе матрица $F\left(X_{1} X_{2}^{-1}\right)$ является диагональной. Вычислим ее ненулевые матричные элементы. Подставляя выражения для $A_{i}, B_{i}, C_{i}, D_{i}, i=1,2$, получим, например, для первого уравнения системы (Г.1)

$$
\begin{aligned}
F\left(X_{1} X_{2}^{-1}\right)\left[\left(c_{1} \bar{c}_{1} Z_{1}-b_{1} \bar{b}_{1} u\right)\left(c_{2} \bar{c}_{2} Z_{2}-b_{2} \bar{b}_{2} u\right)-\right. \\
\left.\quad-u\left(b_{1} \bar{d}_{1}-c_{1} \bar{a}_{1} Z_{1}\right) X_{1} X_{2}^{-1}\left(d_{2} \bar{b}_{2}-a_{2} \bar{c}_{2} Z_{2}\right)\right]= \\
=\left[\left(c_{3} \bar{c}_{3} Z_{1}-b_{3} \bar{b}_{3} u\right)\left(c_{4} \bar{c}_{4} Z_{2}-b_{4} \bar{b}_{4} u\right)-\right. \\
\left.\quad-u\left(b_{3} \bar{d}_{3}-c_{3} \bar{a}_{3} Z_{1}\right) X_{1} X_{2}^{-1}\left(d_{4} \bar{b}_{4}-a_{4} \bar{c}_{4} Z_{2}\right)\right] F\left(X_{1} X_{2}^{-1}\right) .
\end{aligned}
$$

Раскрывая скобки, действуя обеими частями этого уравнения на вектор $\left|k_{1}, k_{2}\right\rangle$, приравнивая коэффициенты при линейно независимых векторах и при разных степенях $u$, получим

$$
\begin{gathered}
c_{1} \bar{c}_{1} c_{2} \bar{c}_{2}=c_{3} \bar{c}_{3} c_{4} \bar{c}_{4}, \quad b_{1} \bar{b}_{1} b_{2} \bar{b}_{2}=b_{3} \bar{b}_{3} b_{4} \bar{b}_{4}, \\
b_{1} \bar{d}_{1} d_{2} \bar{b}_{2}=b_{3} \bar{d}_{3} d_{4} \bar{b}_{4}, \quad c_{1} \bar{a}_{1} a_{2} \bar{c}_{2}=c_{3} \bar{a}_{3} a_{4} \bar{c}_{4}, \\
F\left(\omega^{k+1}\right)=\frac{b_{3} \bar{c}_{4}\left(\bar{d}_{3} a_{4} \omega^{k+1}-\bar{b}_{3} c_{4}\right)}{b_{1} \bar{c}_{2}\left(\bar{d}_{1} a_{2} \omega^{k+1}-\bar{b}_{1} c_{2}\right)} F\left(\omega^{k}\right)=\frac{c_{1} \bar{b}_{2}\left(\bar{a}_{1} d_{2} \omega^{k+1}-\bar{c}_{1} b_{2}\right)}{c_{3} \bar{b}_{4}\left(\bar{a}_{3} d_{4} \omega^{k+1}-\bar{c}_{3} b_{4}\right)} F\left(\omega^{k}\right),
\end{gathered}
$$

где $F\left(\omega^{k}\right), k=0, \ldots, N-1,-$ диагональные матричные элементы матришы $F$. Используя полученные выше ограничения на $a_{i}, b_{i}, c_{i}, d_{i}$, сводим эту систему к системе

$$
\begin{gathered}
\bar{c}_{1} c_{2}=\bar{c}_{3} c_{4}, \quad \bar{d}_{1} d_{2}=\bar{d}_{3} d_{4} \\
F\left(\omega^{k+1}\right)=\frac{\bar{d}_{3} a_{4} \omega^{k+1}-\bar{b}_{3} c_{4}}{\bar{d}_{1} a_{2} \omega^{k+1}-\bar{b}_{1} c_{2}} F\left(\omega^{k}\right)=\frac{\bar{a}_{1} d_{2} \omega^{k+1}-\bar{c}_{1} b_{2}}{\bar{a}_{3} d_{4} \omega^{k+1}-\bar{c}_{3} b_{4}} F\left(\omega^{k}\right) .
\end{gathered}
$$


Легко видеть, что второе уравнение можно вывести из первого, используя соотношения

$$
\bar{c}_{3} \bar{d}_{3}=c_{2} d_{2} \frac{\bar{a}_{3} \bar{b}_{3}}{a_{2} b_{2}}, \quad c_{4} d_{4}=\bar{c}_{1} \bar{d}_{1} \frac{a_{2} b_{2}}{\bar{a}_{1} \bar{b}_{1}} .
$$

Поскольку $F\left(\omega^{k}\right)$ - однозначная функция, две дроби, через которые она выражается, необходимо приравнять друг к другу. Поэтому

$$
\left(\bar{d}_{3} a_{4} \omega^{k+1}-\bar{b}_{3} c_{4}\right)\left(\bar{a}_{3} d_{4} \omega^{k+1}-\bar{c}_{3} b_{4}\right)=\left(\bar{d}_{1} a_{2} \omega^{k+1}-\bar{b}_{1} c_{2}\right)\left(\bar{a}_{1} d_{2} \omega^{k+1}-\bar{c}_{1} b_{2}\right) .
$$

Приравнивая коэффициенты при разных степенях $\omega$, найдем

$$
\begin{gathered}
\bar{a}_{1} \bar{d}_{1} a_{2} d_{2}=\bar{a}_{3} \bar{d}_{3} a_{4} d_{4}, \quad \bar{b}_{1} \bar{c}_{1} b_{2} c_{2}=\bar{b}_{3} \bar{c}_{3} b_{4} c_{4} \\
\bar{a}_{1} \bar{b}_{1} a_{2} b_{2}\left(\frac{\bar{c}_{1} \bar{d}_{1}}{\bar{a}_{1} \bar{b}_{1}}+\frac{c_{2} d_{2}}{a_{2} b_{2}}\right)=a_{4} b_{4} \bar{a}_{3} \bar{b}_{3}\left(\frac{\bar{c}_{3} \bar{d}_{3}}{\bar{a}_{3} \bar{b}_{3}}+\frac{c_{4} d_{4}}{a_{4} b_{4}}\right) .
\end{gathered}
$$

Если опять учесть ограничения на $a_{i}, b_{i}, c_{i}, d_{i}$, то отсюда получим

$$
\bar{c}_{1} c_{2}=\bar{c}_{3} c_{4},
$$

т.е. то же, что и раньше. Кроме того, из очевидного равенства

$$
F\left(\omega^{N+k}\right)=F\left(\omega^{k}\right)
$$

следует, что

$$
\bar{c}_{1}^{N} b_{2}^{N}-\bar{a}_{1}^{N} d_{2}^{N}=\bar{c}_{3}^{N} b_{4}^{N}-\bar{a}_{3}^{N} d_{4}^{N} .
$$

Покажем, что формулы (Г.2) и (Г.3) следуют из формул (33)-(37). Имеем

$$
\begin{aligned}
& \psi_{3}=a_{3}^{N} \bar{c}_{3}^{N}=\frac{c_{2}^{N} \bar{b}_{1}^{N} a_{1}^{N}}{b_{2}^{N}} \frac{b_{2}^{N} \bar{c}_{1}^{N}-\bar{a}_{1}^{N} d_{2}^{N}}{c_{2}^{N} \bar{b}_{1}^{N}-a_{2}^{N} \bar{d}_{1}^{N}} \\
& \phi_{4}=d_{4}^{N} \bar{b}_{4}^{N}=\frac{\bar{d}_{1}^{N} a_{2}^{N} \bar{b}_{2}^{N}}{\bar{a}_{1}^{N}} \frac{b_{2}^{N} \bar{c}_{1}^{N}-\bar{a}_{1}^{N} d_{2}^{N}}{c_{2}^{N} \bar{b}_{1}^{N}-a_{2}^{N} \bar{d}_{1}^{N}} .
\end{aligned}
$$

Выражая отсюда $\bar{c}_{3}^{N}$ и $d_{4}^{N}$ и подставляя в $(Г .3)$, получим тождественное равенство. Кроме того, имеем

$$
\frac{\bar{c}_{3}}{d_{4}}=\frac{c_{2}}{\bar{d}_{1}} \frac{\bar{a}_{1} \bar{b}_{1}}{a_{2} b_{2}}
$$

Умножая последнее уравнение на

$$
c_{4} d_{4}=\bar{c}_{1} \bar{d}_{1} \frac{a_{2} b_{2}}{\bar{a}_{1} \bar{b}_{1}}
$$

получим (Г.2). Таким образом,

$$
F\left(\omega^{k+1}\right)=\sqrt[N]{\frac{\bar{d}_{1}^{N} a_{2}^{N}-\bar{b}_{1}^{N} c_{2}^{N}}{\bar{a}_{1}^{N} d_{2}^{N}-\bar{c}_{1}^{N} b_{2}^{N}}} \frac{\bar{c}_{1} b_{2}-\bar{a}_{1} d_{2} \omega^{k+1}}{\bar{b}_{1} c_{2}-\bar{d}_{1} a_{2} \omega^{k+1}} F\left(\omega^{k}\right)
$$


ПРИЛОЖЕНИЕ Д

\section{Уравнение "звезда-треугольник"}

Здесь мы докажем соотношение "звезда-треугольник"

$G\left(\tilde{q}, \tilde{p} ; Z_{1}\right) F\left(\tilde{p}, r ; X_{1} X_{2}^{-1}\right) G\left(p, q ; Z_{1}\right)=\mu F\left(p^{\prime}, q^{\prime} ; X_{1} X_{2}^{-1}\right) G\left(p, r^{\prime} ; Z_{1}\right) F\left(q, r ; X_{1} X_{2}^{-1}\right)$

и найдем $\mu$. Имеем следуюшую диаграмму:

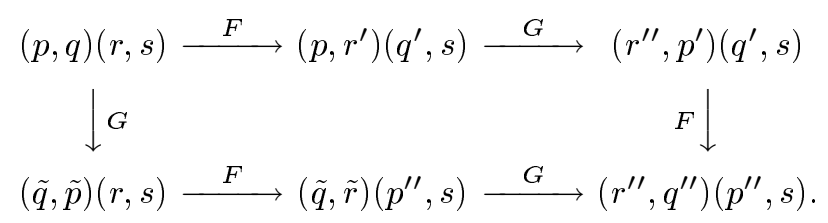

Если параметры $r^{\prime \prime}, q^{\prime \prime}, p^{\prime \prime}$, полученные двумя разными способами, совпадут между собой с точностью до калибровочных преобразований, то равенство (Д.1) действительно будет иметь место.

Напомним определения функций $\Lambda\left(p_{1}, p_{2}\right)$ и $\Omega\left(p_{1}, p_{2}\right)$ :

$$
\Lambda\left(p_{1}, p_{2}\right)=\sqrt[N]{\frac{b_{1}^{N} d_{2}^{N}-c_{1}^{N} a_{2}^{N}}{d_{1}^{N} b_{2}^{N}-a_{1}^{N} c_{2}^{N}}}, \quad \Omega\left(p_{1}, p_{2}\right)=\sqrt[N]{\frac{c_{1}^{N} b_{2}^{N}-a_{1}^{N} d_{2}^{N}}{b_{1}^{N} c_{2}^{N}-d_{1}^{N} a_{2}^{N}}} .
$$

Проведем вычисления для первой цепочки рассматриваемой диаграммы. Для $(p, q)(r, s) \stackrel{F}{\longrightarrow}\left(p, r^{\prime}\right)\left(q^{\prime}, s\right)$ имеем

$$
\begin{array}{ll}
a_{r}^{\prime}=a_{r}, & a_{q}^{\prime}=a_{q}, \\
b_{r}^{\prime}=b_{r}, & b_{q}^{\prime}=b_{q}, \\
c_{r}^{\prime}=\Omega(q, r) c_{r}, & c_{q}^{\prime}=\Omega(q, r)^{-1} c_{q}, \\
d_{r}^{\prime}=\Omega(q, r)^{-1} d_{r}, & d_{q}^{\prime}=\Omega(q, r) d_{q} .
\end{array}
$$

Для $\left(p, r^{\prime}\right)\left(q^{\prime}, s\right) \stackrel{G}{\longrightarrow}\left(r^{\prime \prime}, p^{\prime}\right)\left(q^{\prime}, s\right)$ имеем

$$
\begin{array}{ll}
a_{r}^{\prime \prime}=a_{r}^{\prime}, & a_{p}^{\prime}=a_{p}, \\
b_{r}^{\prime \prime}=b_{r}^{\prime}, & b_{p}^{\prime}=b_{p}, \\
c_{r}^{\prime \prime}=\Lambda\left(p, r^{\prime}\right) c_{r}^{\prime}, & c_{p}^{\prime}=\Lambda\left(p, r^{\prime}\right)^{-1} c_{p}, \\
d_{r}^{\prime \prime}=\Lambda\left(p, r^{\prime}\right)^{-1} d_{r}^{\prime}, & d_{p}^{\prime}=\Lambda\left(p, r^{\prime}\right) d_{p} .
\end{array}
$$

Для $\left(r^{\prime \prime}, p^{\prime}\right)\left(q^{\prime}, s\right) \stackrel{F}{\longrightarrow}\left(r^{\prime \prime}, q^{\prime \prime}\right)\left(p^{\prime \prime}, s\right)$ имеем

$$
\begin{array}{ll}
a_{q}^{\prime \prime}=a_{q}^{\prime}, & a_{p}^{\prime \prime}=a_{p}^{\prime}, \\
b_{q}^{\prime \prime}=b_{q}^{\prime}, & b_{p}^{\prime \prime}=b_{p}^{\prime}, \\
c_{q}^{\prime \prime}=\Omega\left(p^{\prime}, q^{\prime}\right) c_{q}^{\prime}, & c_{p}^{\prime \prime}=\Omega\left(p^{\prime}, q^{\prime}\right)^{-1} c_{p}^{\prime}, \\
d_{q}^{\prime \prime}=\Omega\left(p^{\prime}, q^{\prime}\right)^{-1} d_{q}^{\prime}, & d_{p}^{\prime \prime}=\Omega\left(p^{\prime}, q^{\prime}\right) d_{p}^{\prime} .
\end{array}
$$


Теперь проведем вычисления для второй цепочки диаграммы.

Для $(p, q)(r, s) \stackrel{G}{\longrightarrow}(\tilde{q}, \tilde{p})(r, s)$ имеем

$$
\begin{array}{ll}
\tilde{a}_{q}=a_{q}, & \tilde{a}_{p}=a_{p}, \\
\tilde{b}_{q}=b_{q}, & \tilde{b}_{p}=b_{p}, \\
\tilde{c}_{q}=\Lambda(p, q) c_{q}, & \tilde{c}_{p}=\Lambda(p, q)^{-1} c_{p}, \\
\tilde{d}_{q}=\Lambda(p, q)^{-1} d_{q}, & \tilde{d}_{p}=\Lambda(p, q) d_{p} .
\end{array}
$$

Для $(\tilde{q}, \tilde{p})(r, s) \stackrel{F}{\longrightarrow}(\tilde{q}, \tilde{r})\left(p^{\prime \prime}, s\right)$ имеем

$$
\begin{array}{llrl}
\tilde{a}_{r} & =a_{r}, & & a_{p}^{\prime \prime}=\tilde{a}_{p}, \\
\tilde{b}_{r} & =b_{r}, & b_{p}^{\prime \prime} & =\tilde{b}_{p}, \\
\tilde{c}_{r} & =\Omega(\tilde{p}, r) c_{r}, & c_{p}^{\prime \prime} & =\Omega(\tilde{p}, r)^{-1} \tilde{c}_{p}, \\
\tilde{d}_{r} & =\Omega(\tilde{p}, r)^{-1} d_{r}, & d_{p}^{\prime \prime} & =\Omega(\tilde{p}, r) \tilde{d}_{p} .
\end{array}
$$

Для $(\tilde{q}, \tilde{r})\left(p^{\prime \prime}, s\right) \stackrel{G}{\longrightarrow}\left(r^{\prime \prime}, q^{\prime \prime}\right)\left(p^{\prime \prime}, s\right)$ имеем

$$
\begin{array}{ll}
a_{r}^{\prime \prime}=\tilde{a}_{r}, & a_{q}^{\prime \prime}=\tilde{a}_{q}, \\
b_{r}^{\prime \prime}=\tilde{b}_{r}, & b_{q}^{\prime \prime}=\tilde{b}_{q}, \\
c_{r}^{\prime \prime}=\Lambda(\tilde{q}, \tilde{r}) \tilde{c}_{r}, & c_{q}^{\prime \prime}=\Lambda(\tilde{q}, \tilde{r})^{-1} \tilde{c}_{q}, \\
d_{r}^{\prime \prime}=\Lambda(\tilde{q}, \tilde{r})^{-1} \tilde{d}_{r}, & d_{q}^{\prime \prime}=\Lambda(\tilde{q}, \tilde{r}) \tilde{d}_{q} .
\end{array}
$$

Остается проверить, что полученные $L$-операторы действительно совпадают. Сравнивая параметры $r^{\prime \prime}, q^{\prime \prime}, p^{\prime \prime}$, полученные двумя разными способами, заключаем, что равенство (41) выполняется, если

$$
\begin{aligned}
\Lambda\left(p, r^{\prime}\right) \Omega\left(p^{\prime}, q^{\prime}\right) & =\Lambda(p, q) \Omega(\tilde{p}, r), \\
\Omega(q, r) \Lambda\left(p, r^{\prime}\right) & =\Omega(\tilde{p}, r) \Lambda(\tilde{q}, \tilde{r}) .
\end{aligned}
$$

Нетрудно показать, что равенства (Д.2) выполняются тождественно.

Таким образом, мы доказали соотношение (Д.1) для некоторого, пока неизвестного параметра $\mu$. Найдем $\mu^{N}$. Очевидно, что

$$
\mu^{N}=\frac{\operatorname{det} G(p, q) \operatorname{det} F(\tilde{p}, r) \operatorname{det} G(\tilde{q}, \tilde{r})}{\operatorname{det} F(q, r) \operatorname{det} G\left(p, r^{\prime}\right) \operatorname{det} F\left(p^{\prime}, q^{\prime}\right)} .
$$

Детерминанты матриц $G\left(p_{1}, p_{2}\right)$ и $F\left(p_{1}, p_{2}\right)$ нетрудно найти. Каждую из них можно по отдельности привести к диагональному виду (не одновременно), причем в каждом случае диагональные матричные элементы даются следующими формулами:

$$
\begin{aligned}
& \frac{G\left(p_{1}, p_{2} ; \omega^{k}\right)}{G\left(p_{1}, p_{2} ; 1\right)}=\left(\frac{b_{1}^{N} d_{2}^{N}-c_{1}^{N} a_{2}^{N}}{d_{1}^{N} b_{2}^{N}-a_{1}^{N} c_{2}^{N}}\right)^{\frac{k}{N}} \prod_{j=1}^{k} \frac{d_{1} b_{2}-a_{1} c_{2} \omega^{j}}{b_{1} d_{2}-c_{1} a_{2} \omega^{j}}, \\
& \frac{F\left(p_{1}, p_{2} ; \omega^{k}\right)}{F\left(p_{1}, p_{2} ; 1\right)}=\left(\frac{b_{1}^{N} c_{2}^{N}-d_{1}^{N} a_{2}^{N}}{c_{1}^{N} b_{2}^{N}-a_{1}^{N} d_{2}^{N}}\right)^{\frac{k}{N}} \prod_{j=1}^{k} \frac{c_{1} b_{2}-a_{1} d_{2} \omega^{j}}{b_{1} c_{2}-d_{1} a_{2} \omega^{j}} .
\end{aligned}
$$


Положим

$$
G\left(p_{1}, p_{2} ; 1\right)=F\left(p_{1}, p_{2} ; 1\right)=1 \text {. }
$$

Тогда

$$
\begin{aligned}
& \operatorname{det} G\left(p_{1}, p_{2}\right)=\left(\frac{b_{1}^{N} d_{2}^{N}-c_{1}^{N} a_{2}^{N}}{d_{1}^{N} b_{2}^{N}-a_{1}^{N} c_{2}^{N}}\right)^{\frac{N-1}{2}} \prod_{k=1}^{N-1} \prod_{j=1}^{k} \frac{d_{1} b_{2}-a_{1} c_{2} \omega^{j}}{b_{1} d_{2}-c_{1} a_{2} \omega^{j}} \\
& \operatorname{det} F\left(p_{1}, p_{2}\right)=\left(\frac{b_{1}^{N} c_{2}^{N}-d_{1}^{N} a_{2}^{N}}{c_{1}^{N} b_{2}^{N}-a_{1}^{N} d_{2}^{N}}\right)^{\frac{N-1}{2}} \prod_{k=1}^{N-1} \prod_{j=1}^{k} \frac{c_{1} b_{2}-a_{1} d_{2} \omega^{j}}{b_{1} c_{2}-d_{1} a_{2} \omega^{j}}
\end{aligned}
$$

ПРИЛОЖКЕНИЕ Е

\section{Соотношения в алгебре $Q$-операторов}

Соотношения в алгебре $Q$-операторов следуют из свойств циклических представлений алгебры $L$-операторов. Покажем это.

Соотношения (42)-(45) становятся очевидными, если вспомнить, что

$$
Q(u)=\operatorname{tr}_{0} L_{10}(u) L_{20}(u) \ldots L_{n 0}(u)
$$

и имеют место симметрии

$$
\begin{gathered}
L\left(\lambda p_{1}, \bar{p}_{1}\right)=\lambda L\left(p_{1}, \bar{p}_{1}\right), \\
L\left(p_{1}, \mu \bar{p}_{1}\right)=\mu L\left(p_{1}, \bar{p}_{1}\right), \\
L\left(a_{1}, b_{1}, c_{1}, d_{1}, \bar{a}_{1}, \bar{b}_{1}, \bar{c}_{1}, \bar{d}_{1}\right)=L\left(\lambda a_{1}, b_{1}, \lambda c_{1}, d_{1}, \lambda^{-1} \bar{a}_{1}, \bar{b}_{1}, \lambda^{-1} c_{q}, \bar{d}_{1}\right), \\
L\left(a_{1}, b_{1}, c_{1}, d_{1}, \bar{a}_{1}, \bar{b}_{1}, \bar{c}_{1}, \bar{d}_{1}\right)=L\left(a_{1}, \mu b_{1}, c_{1}, \mu d_{1}, \bar{a}_{1}, \mu^{-1} \bar{b}_{1}, \bar{c}_{1}, \mu^{-1} \bar{d}_{1}\right),
\end{gathered}
$$

где $\lambda, \mu$ - произвольные числа. Соотношение (47) следует из симметрии

$$
L_{1}\left(p_{1}, \bar{p}_{1}\right) L_{2}\left(p_{2}, s\right)=L_{1}\left(p_{1}, \bar{p}_{1}\right) M M^{-1} L_{2}\left(p_{2}, s\right),
$$

или, записывая более подробно,

$$
\begin{aligned}
& L_{1}\left(a_{1}, b_{1}, c_{1}, d_{1}, \bar{a}_{1}, \bar{b}_{1}, \bar{c}_{1}, \bar{d}_{1}\right) L_{2}\left(a_{2}, b_{2}, c_{2}, d_{2}, \bar{a}_{2}, \bar{b}_{2}, \bar{c}_{2}, \bar{d}_{2}\right)= \\
& \quad=L_{1}\left(a_{1}, b_{1}, c_{1}, d_{1}, \beta \bar{a}_{1}, \alpha \bar{b}_{1}, \alpha \bar{c}_{1}, \beta \bar{d}_{1}\right) L_{2}\left(\beta^{-1} a_{2}, \alpha^{-1} b_{2}, \alpha^{-1} c_{2}, \beta^{-1} d_{2}, \bar{a}_{2}, \bar{b}_{2}, \bar{c}_{2}, \bar{d}_{2}\right),
\end{aligned}
$$

где $\alpha, \beta$ - произвольные числа. Здесь матрица

$$
M=\left(\begin{array}{ll}
\alpha & 0 \\
0 & \beta
\end{array}\right) .
$$

Соотношение (46) получается из равенства

$$
G L\left(a_{1}, b_{1}, c_{1}, d_{1}, \bar{a}_{1}, \bar{b}_{1}, \bar{c}_{1}, \bar{d}_{1}\right) G^{-1}=L\left(a_{1}, b_{1}, \Lambda c_{1}, \Lambda^{-1} d_{1}, \bar{a}_{1}, \bar{b}_{1}, \Lambda^{-1} \bar{c}_{1}, \Lambda \bar{d}_{1}\right)
$$


где

$$
\Lambda=\Lambda\left(p_{1}, \bar{p}_{1}\right)=\sqrt[N]{\frac{b_{1}^{N} \bar{d}_{1}^{N}-c_{1}^{N} \bar{a}_{1}^{N}}{d_{1}^{N} \bar{a}_{1}^{N}-a_{1}^{N} \bar{c}_{1}^{N}}},
$$

а соотношение (48) - из равенства

$$
\begin{aligned}
& F L_{1}\left(a_{1}, b_{1}, c_{1}, d_{1}, \bar{a}_{1}, \bar{b}_{1}, \bar{c}_{1}, \bar{d}_{1}\right) L_{2}\left(a_{2}, b_{2}, c_{2}, d_{2}, \bar{a}_{2}, \bar{b}_{2}, \bar{c}_{2}, \bar{d}_{2}\right) F^{-1}= \\
& \quad=L_{1}\left(a_{1}, b_{1}, c_{1}, d_{1}, a_{2}, b_{2}, \Omega c_{2}, \Omega^{-1} d_{2}\right) L_{2}\left(\bar{a}_{1}, \bar{b}_{1}, \Omega^{-1} \bar{c}_{1}, \Omega \bar{d}_{1}, \bar{a}_{2}, \bar{b}_{2}, \bar{c}_{2}, \bar{d}_{2}\right),
\end{aligned}
$$

где

$$
\Omega=\Omega\left(\bar{p}_{1}, p_{2}\right)=\sqrt[N]{\frac{\bar{c}_{1}^{N} b_{2}^{N}-\bar{a}_{1}^{N} d_{2}^{N}}{\bar{b}_{1}^{N} c_{2}^{N}-\bar{d}_{1}^{N} a_{2}^{N}}} .
$$

Благодарности. Мы благодарны Б. Фейгину и Ю. Строганову за полезные обсуждения. Один из нас (А.О.) благодарен В. Бажанову за обсуждение свойства факторизации $R$-матрищы киральной модели Поттса. Работа выполнена при поддержке следуюших грантов: РФФИ (№ 01-02-16686; № 99-01-01169; № 00-15-96579), CRDF-RP1 № 2254 , INTAS № 00-00055.

\section{Список литературы}

[1] R. J. Baxter. Ann. Phys. 1972. V. 70. P. 193; P. 323; 1973. V. 76. P. 48.

[2] R.J. Baxter, J.H.H. Perk, H. Au-Yang. Phys. Lett. A. 1988. V. 128. P. 138.

[3] H. Au-Yang, B. M. McCoy, J. H. H. Perk, S. Tang, M. L. Yan. Phys Lett. A. 1987. V. 123. P. 219.

[4] R. J. Baxter, V. V. Bazhanov, J. H. H. Perk. Int. J. Mod. Phys. B. 1990. V. 4. № 5. P. 803.

[5] V. V. Bazhanov, Yu. G. Stroganov. J. Stat. Phys. 1990. V. 51. P. 799.

[6] V. O. Tarasov. Int. J. Mod. Phys. A. 1992. V. 7. 1B. P. 963.

[7] V. Pasquier, M. Gaudin. J. Phys. A: Math. Gen. 1992. V. 25. P. 5243.

[8] V. B. Kuznetsov, E. K. Sklyanin. J. Phys. A: Math. Gen. 1998. V. 31. P. 2241.

[9] E. K. Sklyanin. Bäcklund transformations and Baxter's $Q$-operator. nlin.SI/0009009.

[10] G. P. Pronko. Commun. Math. Phys. 2000. V. 212. P. 687.

[11] П. П. Кулиш, Н. Ю. Решетихин. Записки научных семинаров ЛОМИ. 1980. Т. 101. С. 101; P. P. Kulish, N. Yu. Reshetikhin. J. Soviet Math. 1983. V. 23. P. 2435.

[12] M. Jimbo. Lett. Math. Phys. 1986. V. 11. P. 247.

[13] M. Jimbo. Topics from Representations of $U_{q}(\{g\})$ - An Introductory Guide to Physicists. Preprint Kyoto University, Japan, 1991.

[14] T. Deguchi, K. Fabricius, B. M. McCoy. J. Stat. Phys. 2001. V. 102. P. 701; cond-mat/9912141.

[15] S. Pakuliak, S. Sergeev. Relativistic Toda chain at root of unity II. Modified Q-operator. nlin.SI/0107062; C. М. Сергеев. Вполне интегрируемые дискретные системы в трех измерениях. Дисс. д.ф̆.-м.н. Дубна: ОИЯИ, 2001. 